\title{
Association and Content-Based Retrieval
}

\author{
Chabane Djeraba, Member, IEEE Computer Society
}

\begin{abstract}
In spite of important efforts in content-based indexing and retrieval during these last years, seeking relevant and accurate images remains a very difficult query. In the state-of-the-art approaches, the retrieval task may be efficient for some queries in which the semantic content of the query can be easily translated into visual features. For example, finding images of fires is simple because fires are characterized by specific colors (yellow and red). However, it is not efficient in other application fields in which the semantic content of the query is not easily translated into visual features. For example, finding images of birds during migrations is not easy because the system has to understand the query semantic. In the query, the basic visual features may be useful (a bird is characterized by a texture and a color), but they are not sufficient. What is missing is the generalization capability. Birds during migrations belong to the same repository of birds, so they share common associations among basic features (e.g., textures and colors) that the user cannot specify explicitly. In this paper, we present an approach that discovers hidden associations among features during image indexing. These associations discriminate image repositories. The best associations are selected on the basis of measures of confidence. To reduce the combinatory explosion of associations, because images of the database contain very large numbers of colors and textures, we consider a visual dictionary that group together similar colors and textures. Thus, the visual dictionary summarizes the image features. An algorithm based on a clustering strategy creates the visual dictionary. The associations discovered permit the automatic classification of images during their insertion into image repositories and return accurate and relevant results. More generally, we show that content and knowledge-based indexing and retrieval is more efficient than retrieval approaches based on content exclusively and inaugurate a new generation of approaches in which knowledge contributes to finding images in large image repositories.
\end{abstract}

Index Terms_-Image, indexing, retrieval, similarity, association.

\section{INTRODUCTION}

$\mathrm{H}$ ow will the users index the vast and growing repositories of information at their disposal? And, how will the users access the multiple types of digital formats in which their information is stored, including images? They need intelligent tools to sort and organize the onslaught of digital information and to transform that information into usable knowledge.

Knowledge is precise information that meets a need, answers a question, or solves a problem. Knowledgecontent-based retrieval, then, is the ability to access and extract only the most accurate and relevant information. Conventional tools like search engines provide only rudimentary extraction of raw information that, after a time-consuming process of evaluation, all too often turns out to be mostly inaccurate, irrelevant, or incomplete. Reaching and utilizing true knowledge can make the indexing succeed where others fail.

In the current state of the art [18], [11], [8], [13], the automatic aspect of indexing, which is an important advantage of visual information systems, supports weak knowledge description and, therefore, weak query results. Queries based on content are not powerful enough to specify knowledge queries, such as "finding images of flowers in front of a lake" or "finding images of a naval fleet or a shuttle flight during a launch." Such queries are not

- The author is with IRIN, Nantes University, 2, rue de la Houssiniére, 44322 Nantes Cedex, France. E-mail: djeraba@irin.univ-nantes.fr.

Manuscript received 29 Nov. 1999; revised 2 Oct. 2000; accepted 15 June 2001.

For information on obtaining reprints of this article, please send e-mail to: tkde@computer.org, and reference IEEECS Log Number 110998. easy because the associations between the content and the semantic in the user's mind are weakly declared. There is a difference between the image features of the examples (e.g., color, texture) and the semantic (e.g., flowers in front of a lake) the user is looking for. It is not easy because the user has to define the semantic he is looking for in terms of visual descriptions. In the query, the features mentioned earlier may be useful (a shuttle has a special shape, launch has a specific color-orange), but they are not sufficient. What is missing is the generalization capability. A fleet is a grouping of a number of significant regions that correspond to ships sharing common features (e.g., shapes). "Flowers in front of a lake" have specific colors, textures and associations between visual features that are very difficult to specify explicitly in the query. These associations are hidden. The user's mind discriminates "images that contain flowers in front of a lake" from "images that contain flowers in mountains." This is possible because there are several hidden associations between visual features that compose and discriminate the two repositories of images: "flowers in a front of the lake" and "flowers in mountains."

Without efficient indexing, there is no efficient retrieval. We cannot seek the images efficiently without an efficient way of indexing the content of images. The central question is: how can the system extract not only the classical features (e.g., colors, textures), but also the hidden associations between the features in order to make the content-based indexing and retrieval accurate? The answer to this challenge inaugurates a new generation of information retrieval systems in which the knowledge is extracted automatically, and is well positioned to drive the retrieval process efficiently. 
A compromise and a natural way to deal with this challenge seem to obtain some semantic information through manual annotations. We obtain indexing and retrieval methods that combine textual and visual features. Based on experimentation, it is proven that queries based on textual and visual descriptions are more efficient than queries based on textual or (exclusive) visual queries [3], [7]. Visual queries are extracted automatically, however, textual descriptions are annotated manually. Although the effort in "manual" annotation is less important than in classical information retrieval [21], [20], the "manual" property of the annotation is a serious "speed limit" to the exploitation of such approach. Furthermore, the manual annotation is quite subjective and ambiguous and it is very difficult to capture the visual content of an image using words.

One approach to deal with this problem is to organize the digital repositories in a meaningful manner using image classification. Image classification classifies images into semantic repositories that are manually precategorized. The classification of images into repositories can be helpful in the semantic organization of digital repositories. The classification of images is quite difficult in general. Images in the same semantic repositories may have large variations with dissimilar visual descriptions (images of persons, images of industries, etc.), and images from different semantic repositories might share a common background (some flowers and sunset have similar colors). And, this limits the efficiency of automatic classification based exclusively on visual content of images (texture, color).

In this paper, we propose a new scheme for automatic hierarchical image classification. We assume a training set of images with known repository labels available. We use low-level features-histogram of colors and Fourier descriptors of texture- that are together efficient for contentbased image retrieval. Using low-level features for the training images, associations among visual features are extracted automatically. These associations discriminate image repositories. The best associations are selected on the basis of two confidence measurements (conditional probability and implication intensity). Once the classification tree is obtained, any new image can be classified easily. Furthermore, query results are obtained with semantics because they belong to semantic repositories. We prove that discovering hidden associations contributes to making the content-based retrieval more efficient. The hierarchical approach of image classification has several advantages: easy browsing and navigation through the repositories, efficient retrieval, and ergonomically friendly presentation of repositories.

More generally, in the application context, the proposed approach triggers a solution that transforms information into knowledge. It empowers people and enables organizations to analyze, index, catalog, browse, access, and search more easily all image knowledge assets. Employing simple queries-by-example, the approach is intuitive and easy to use.

In Sections 2 and 3, we present the main contribution of our work and related works. In Section 4, we present the advanced framework of the approach. In Section 5, we present image features. In Section 6, we present how to create visual dictionaries from digital images. In Section 7, we highlight the interest of the knowledge discovery approach, and show how the knowledge discovery engine is used to learn discriminating characteristics that are fundamental to classification. In Section 8, we present result evaluations.

\section{Contribution}

The scope of the proposed approach concerns the automatic discovery of hidden associations. Automatic discovery of hidden associations proposes a new framework for knowledge discovery by enabling the extraction of associations between visual features that contribute to identifying and understanding image repositories. These capture the discriminations of different repositories of images, such as the naval fleet or flowers in front of a lake.

The most difficult problem we met using the association discovery method among visual features is to find features that are shared by images. Without a clustering step in which similar features are grouped together, we will obtain different features for different images, even if they are similar. So, for 1.000.000 images, we will have 1.000.000 colors and 1.000.000 textures. In such cases, the discovery method returns image specific associations that are completely inefficient in discriminating repositories of images. For these reasons, we developed an approach that group together similar features (colors and textures). For example, in the association $a=>b, a$, and $b$ are centroids of similar colors or textures. So, $a$ and $b$ are features that may be found in several images.

The approach developed is articulated around two important points:

First, the definition of a clustering algorithm for image descriptors (histogram of colors and Fourier descriptors of textures). The algorithm is an extension of k-means [17] by image descriptors and suitable similarity measures (quadratic distance). The clustering algorithm creates an efficient visual dictionary that summarizes the database image features. The visual dictionary is a fundamental step for discriminating features necessary to knowledge extraction. The visual dictionary groups together similar features based on the clustering algorithm. The visual dictionary tends to maximum entropy and minimum distortion.

Second, the power confidence values of the associations among visual features. They are composed of conditional probability and implication intensity measures.

The implementation of these notions, together in the same framework, constitutes our knowledge-content-based indexing that extends the classical indexing, limited to visual features, by relevant associations extracted automatically.

The proposed approach learns application domain associations automatically and contributes to extracting the knowledge from image repositories. Users may reuse the learned knowledge easily and naturally in the browsing process. Furthermore, there is no need for "a priori" knowledge in order to have efficient access to information. So, the system is generic and adaptable to any application field. The system discovers knowledge in images without predetermined knowledge about the application field. 


\section{Related Works}

Very few studies have considered knowledge extraction and data classification on the basis of image features, and very few of them, [12], [2], concern knowledge extraction and data classification in the context of image indexing and retrieval. That is why we will consider the related work in the general context of information retrieval in different repositories of images.

In the general context of information retrieval, the majority of the related work has been concerned with handling text information. Some research used a main index for repository selection. The main index supports specification of all repositories. The content of repositories are described by a set of attributes. The selection of appropriate repositories is trivial. The retrieval process starts in the main index that provides users directions on repositories to be searched. In this category of research, we find systems such as Wide Area Information server [14], Indie server [4], and the World Wide Web search engines (ALIWEB [16], WebSeek [1], AltaVista, Yahoo, etc.). If ALIWEB maintains a manually generated index for each repository, AltaVista and Yahoo maintain an automaticgenerated index. The Web image search engine, WebSeek, uses a hierarchical semantic structure for collecting and searching images from the Web. The image categories and hierarchy are preset by human design. An image is classified into one of the repositories by first extracting key words from html pages and then mapping the key words to repositories.

With the high popularity and increasing volume of images in centralized and distributed environment, it is evident that the repository selection methods based on textual description is not suitable for visual queries, where the user's queries may be unanticipated and referring to unextracted image content. Furthermore, they require human assistance and depend on the information in the HTML pages that may be insufficient or even inaccurate and misleading.

More recently, a method for data resource selection in distributed visual information systems [2], has been proposed. The method is based on a metadata base at a query distribution server. The metadata base records a summary of the visual content of the images in each repository through image templates and statistical features. Image templates and statistical features characterize the similarity distributions of the images. The selection of the databases is driven by searching the metadata base using a ranking algorithm that uses query similarity to a template and the features of the database associated with the template. Two selection approaches have been implemented, termed by mean-based and histogram-based approaches. The template summarizes the visual contentbased on basic features (histogram of color and mean of colors). An important difference with our approach concerns the use of mean and histogram of colors; our approach uses histograms of color and Fourier coefficients of textures. Finally, visual templates and statistical features summarize the content of repositories; in our approach, associations among visual features summarize the content of repositories.
Another approach [12] proposes a new scheme for automatic hierarchical image classification. It assumes that a training set of images with known repository labels is available. It uses image descriptors: banded color correlograms. Using banded color correlograms, the approach models the features using singular value decomposition and constructs a classification tree. Once the classification tree is obtained, any new image can be classified. The method has been tested on 11 repositories of COREL collection (aviation, photography, British motor car collection, etc.). The classification tree obtained seems to be conforming to the semantic content of the 11 repositories. An interesting point of this approach is the use of correlograms. The results suggest that correlograms have more latent semantic structures than histograms. The technique used extracts a certain form of knowledge to classify images. Using a noisetolerant SVD [5] description, the image is classified in the training data using the nearest neighbor with the first neighbor dropped. Based on the performance of this classification, the repositories are partitioned in subrepositories, and the interclass dissociation is minimized. This is accomplished using normalized cuts. The subrepositories and the training images that were correctly classified with respect to the subrepositories are worked upon recursively to obtain a hierarchical classification tree.

Compared to this approach [12], our approach uses two basic visual features: histograms of colors and Fourier descriptors of the texture. These two descriptors make the description of the image content accurate. So, our approach extracts automatically the relationships between these two descriptors in each repository of images. The extraction of relationships is not possible in this approach as the content of images are represented by only one descriptor: banded color correlograms.

In the general context of content-based image retrieval, except few cases such as those presented above, the state of the art is weak. Although many visual information systems have been developed recently [8], [18], none of these systems operate by considering knowledge extracted from image repositories. The selection of image repositories for a given query discussed in this paper offers a new approach to design a knowledge-content-based retrieval system. The proposed approach in our work may also be used for the Web search engines once the image repositories on the Web have been collected, and may be seen as one of the first investigations in knowledge discovery from visual data.

\section{Advanced Framework}

Basically, the framework is composed of two important components: indexing and retrieval. In indexing, the image contents are extracted automatically. Methods, whether aided by the user or not, may identify relevant regions in images and compute features such as color and texture of the regions or compute the visual features of the whole image. The extracted contents are represented as or transformed into suitable models and data structures, and then stored in the database. In an optimal framework, the representation of the contents is managed by a virtual memory, in which the most frequently accessed contents are kept in the high-speed memory (e.g., central memory) 
and the remaining contents are kept in lesser speed memory (e.g., secondary memory). The retrieval consists of searching images by selecting target images or content properties such as color, texture of image regions, or combinations of these. The system includes a visual query tool that lets users form a query by drawing, sketching and selecting textures, and colors. Finally, the retrieval process computes distances between source and target features, and sorts the most similar images. The basic properties of the framework presented so far are classical and shared by lots of visual information systems [8], [13], [11]. In the context of knowledge-content-based indexing and retrieval, we propose a suitable framework in which three extensions are concerned:

First, on the basis of semantic repositories (Birds, Flowers, Water, Buildings, etc.), the system extracts image features and discovers the knowledge (associations shared by images) that discriminates each repository. The associations describe relationships between visual features (color and textures of images). Each set of associations linked to a repository summarizes image contents of the repository. Associations contribute to repository discriminations. The features and knowledge extracted are saved in the database.

Second, when an image is inserted into the database, it is classified "automatically" in the repository hierarchy. At the end of the classification process, the image is added to a specific repository. In this case, the distance between the image and the knowledge associated to the repository is the shortest one, compared to the distance between the image and the other repositories. Otherwise, the instantiation association between the image and the repository is not considered.

Third, the architecture supports efficient retrievals and browsing through repositories. For example, the user may ask queries such as "find images similar to the source image but only in People repositories" or "find all images that illustrate the bird repository with such colors and such texture." In the retrieval task, when the final repository is selected, features (colors, textures) of the query specification are compared with features of the image repository to determine which images correctly match (are similar to) the given features. The matching task is based on computing the distance between target and source image regions. When mixing several features, such as colors and textures, the resulting distance is equal to the Sum taking into account the confidence values of the considered features. The resulting images are sorted; the shortest distance corresponds to the most similar images.

Ideally, we should develop a fully automated system that, after extracting and storing visual features of images, clusters together similar images in repositories. Each repository is automatically obtained and should correspond to a semantic repository of the application field. However, this is not realistic, as some tasks inevitably require the user's intervention such as repository semantic identification and validation.

These extensions improve system performance compared to previous versions [6] of the system. For example, in the retrieval task, when the user gives an example image (called source image) to formulate his query and asks "find images similar to the source image," the system will not match the source image with all images in repositories. It will match the source image features with only the target image features of suited repositories (Fig. 1). If the knowledge that characterizes a repository is globally respected by the source image, then the considered repository is the suited one. Then, the system focuses the search on the subrepositories of the current one. In the target repository, the search is, generally, sequential.

Another advantage of these extensions is the richness of the description contained in the results of queries since the system presents both similar images and their repositories.

For example, the user specifies the query: "Find images that are visually similar to query images." The query images represent waterfalls (Fig. 2). The system matches the query images with both repository centroids and knowledge, in the form of associations of the different repositories. The image belongs to waterfall repository because the distance between the image and the class centroid of the image repository is the shortest one, and the associations associated with the repository are globally verified. The retrieval process, then, matches all the images of this repository in a sequential order. The first images returned contain waterfalls. All the images are visually similar to the example images. This example illustrates "query by examples" that are based on combination of the visual features. "Query by examples" specifies a query that means "find images that are similar to those specified." The query may be composed of several images. Several images make the "efficiency" of retrieval accurate. For example, several images of "waterfall" gives us an accurate description of the waterfall. This property possible enables the refinement of retrieval based on the feedbacks (results of previous queries).

\section{Image Features}

The color is the first feature considered to describe the image content. The color feature is extracted automatically from an image or a region. In the first step of the extraction process, based on the physical format, the region or image color is extracted and represented in the RGB model. Based on the RGB model, the color is transformed into HSV model, characterized by these means $\mathrm{H}, \mathrm{S}$, and V. The HSV model is more suited than the RGB model, in which certain ambiguities appear between colors (e.g., yellow and green).

In the object-oriented modeling, we define a class of colors called HSV. HSV repository includes color histogram and methods (e.g., distance measures). The color of a region is represented by a histogram of 256 colors. Each element of the histogram represents the number of pixels that have the suited color. So, comparing the colors of two regions is equivalent to computing the distance between the histogram of the target and the source regions. Before submitting the query, the user may choose the distance. By default, the quadratic distance is activated.

The texture is an important aspect of human visual perception, and it is the second important feature extracted automatically from image regions. The approach considered implements a powerful texture representation. Thus, we use a mathematical model: Fourier model [22]. The Fourier 


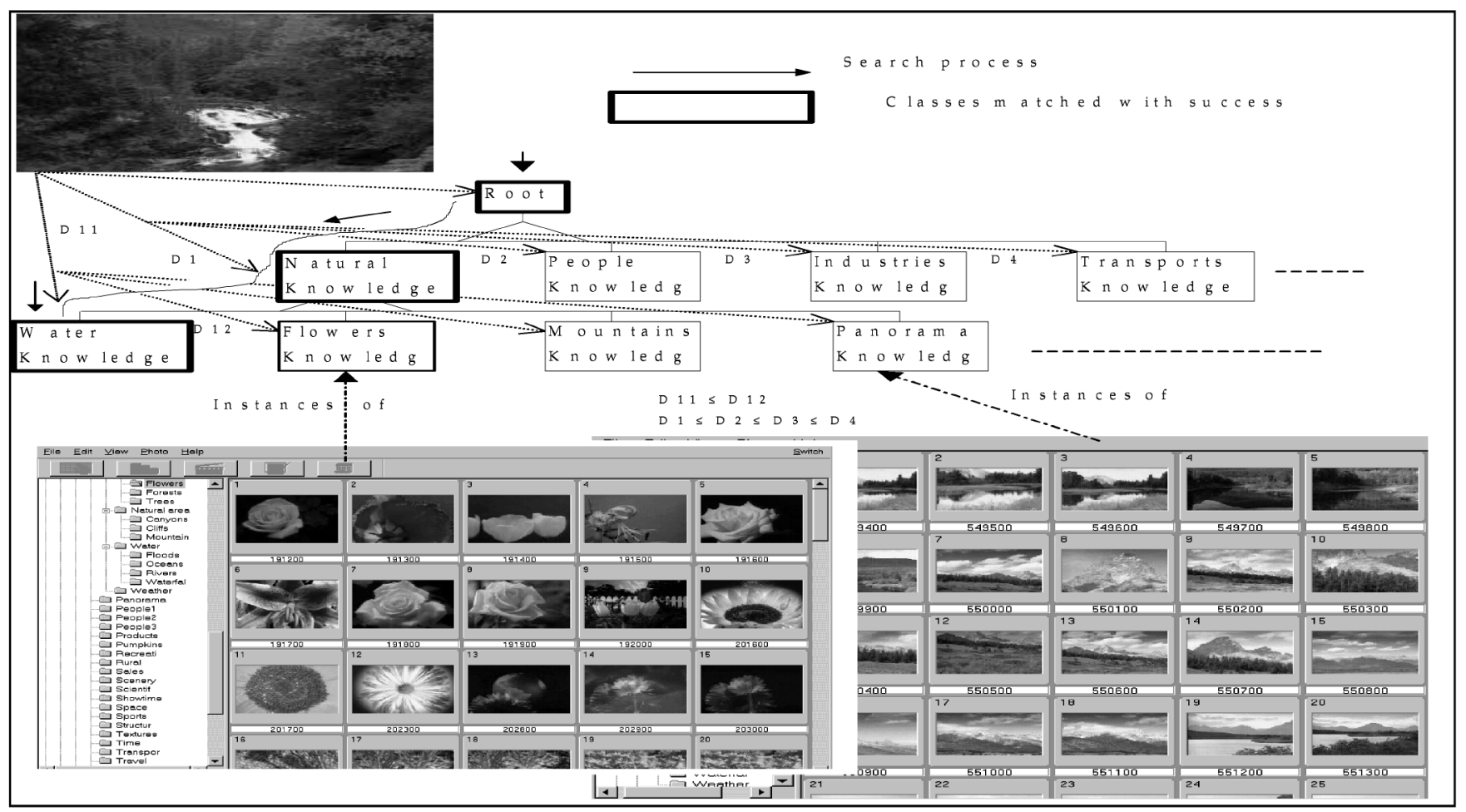

Fig. 1. Example of image retrieval based on knowledge. Screen copies indicate samples of our experimental image repositories. The image repositories zoomed concern, respectively, Panorama and Flowers images.

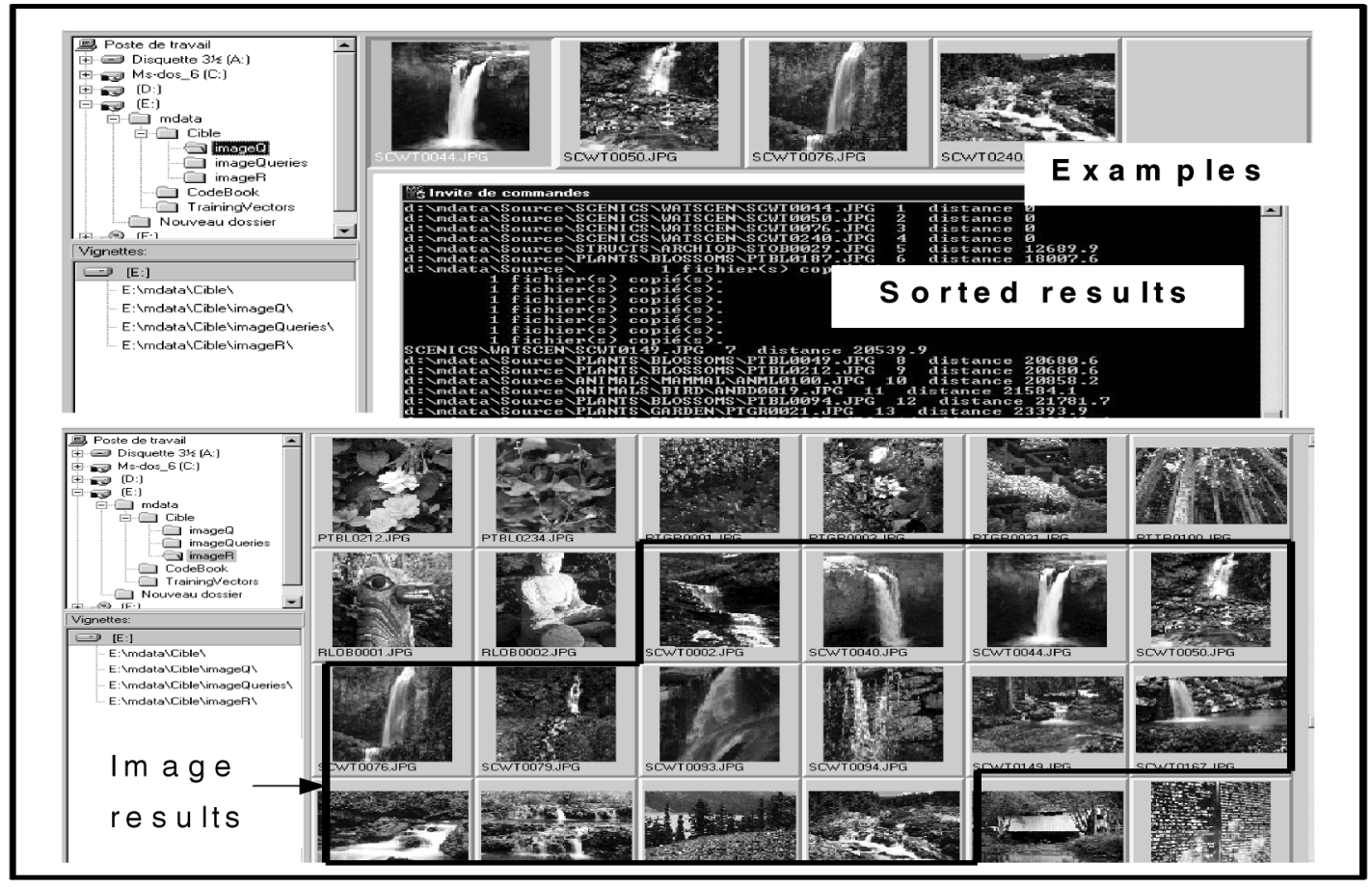

Fig. 2. "Find images that contain waterfalls."

model has very interesting advantages: the texture can be reconstructed from the features. It has a mathematical description rather than a heuristic one. And, finally, the model supports the robustness of description to translation, rotation, and scale transformations. An important contribution of our representation is our extension of Fourier model to texture description. This extension considers the matching process, and particularly the similarity measure. In this extension, we consider texture $(t)$ to be composed of two functions (Fig. 3): $x(t)$ and $y(t)$.

So, texture $(t)=(x(t), y(t)) . x(t)$ represents the different level of gray of $x$, and $y(t)$ represents the different level of gray of $y . t$ indicates the different indices of the signal texture. $t=0, N-1 . N$ is the period of the function and 


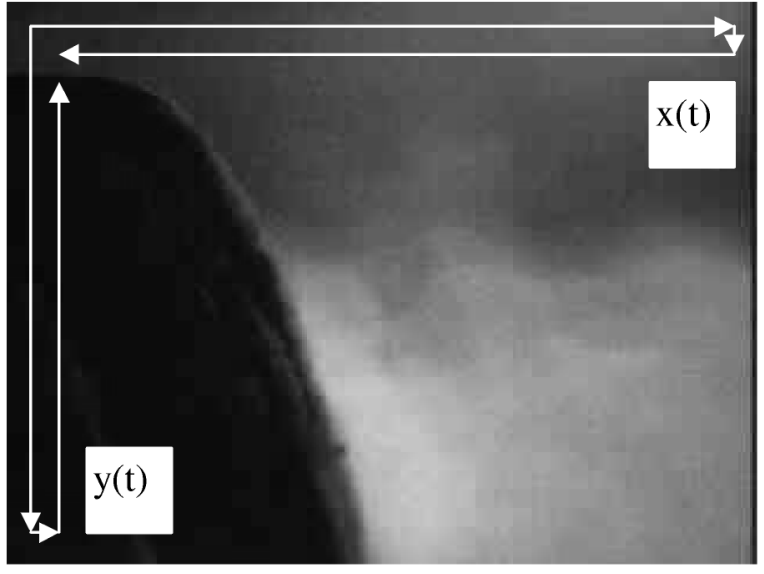

Fig. 3. $x(t), y(t)$.

$\mathrm{N}$ = length of the normalized image. So, we have two series of coefficients $S\left(a_{n}, b_{n}\right)$ and $S\left(c_{n}, d_{n}\right)$ that represent Fourier coefficients of $x(t)$ and $y(t)$, respectively (Fig. 4).

We consider only 11 coefficients of Fourier that select the lowest frequencies of the subband $k \in[0-10]$. In this extension, we modify the similarity measures (Euclidean distance) in order to consider the coefficients of the two signals $x(t)$ and $y(t)$.

The choice of implementing Fourier descriptors is based on its numerous advantages such as texture reconstruction, and robustness of description to translation, rotation, and scale transformations. Furthermore, it represents any complex texture with only few parameters; for $N$ harmonics, we have $2+N * 4$ coefficients.

Two textures are similar even if they differ as a result of a geometric transformation such as rotation, scale, or translation. In fact, translation, rotation, and scale have no effect on the module of Fourier's coefficients (more or less $K$ coefficient, for scale).

\section{Visual Dictionary}

The creation of the visual dictionary is a fundamental preprocessing step necessary to extract associations. It is not possible to extract useful associations without the preprocessing step in which similar features are clustered. The gravity centers of the feature clusters constitute the visual dictionary. Without the visual dictionary, we have to consider all features of all images; then, we obtain very few features shared by images and then very few associations, that discriminate repositories. In such cases, the associations extracted will be too weak to discriminate the repositories.

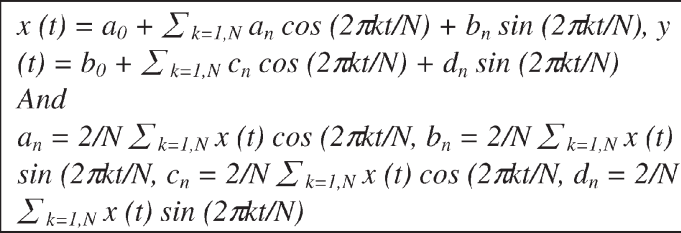

Fig. 4. Fourier texture features.

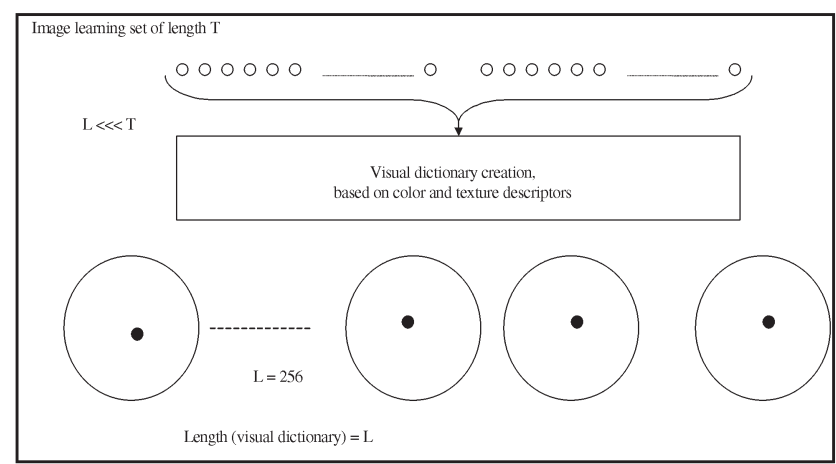

Fig. 5. Clustering and reduction algorithm. Based on the learning set of length equal to $T$ (image repositories contain $T$ images), the algorithm finds a visual dictionary of colors and textures. The length of the visual dictionary is equal to $L$. The visual dictionary contains the most representative colors and textures of images.

Based on the learning set of length equal to $T$ (image repositories contain $T$ images), the algorithm creates a visual dictionary of colors and textures. The length of the visual dictionary is equal to $L$. The visual dictionary contains the most representative colors and textures of images (Fig. 5). In our experiments, $L=256$ and $T=27,331$ images. In other words, the algorithm clusters together similar numerical representations of color and texture. The algorithm result summarizes the color and texture features of the image repositories. The difference between the color and texture clustering algorithms concerns the features (histogram for colors and Fourier coefficients for texture) and distances used, respectively, Euclidean for textures and quadratic for colors.

\subsection{Definitions}

We present definitions of some terms that are useful to understand the principle of the algorithm.

K-Medoid. K-medoid methods determine $k$ cluster representatives and assign each feature to the cluster with its representative closest to the feature such that the sum of the distances squared between the features and their representatives is minimized.

Centroid. The centroid is the gravity center of a set of features. The center of gravity is equal to the mean of feature vectors of a cluster. The centroid

$$
c_{l}=\left(c_{l 1}, c_{l 2}, c_{l 3}, c_{l 4}, \ldots, c_{l n}\right)
$$

is equal to the mean feature vectors

$$
a_{i}=\left(a_{i 1}, a_{i 2}, a_{i 3}, a_{i 4}, \ldots, a_{i n}\right),
$$

$i=[1-m] . m$ is the cardinality of the cluster. $n=$ the length of the vector. $a_{i j}$ is the $j$ th element of the feature $i$. $m=$ the number of features in the cluster. $n=$ the length of a feature. $l \in[1-L], l$ corresponds to the $l$ th cluster. $c_{l}=\left(\sum_{i=1, m} a_{i}\right) / m$, and $c_{l j}=\left(\sum_{i=1, m} a_{i j}\right) / m$. So, the $j$ th element of the centroid vector is equal to the mean of the $j$ th elements of the feature vectors of the cluster, as presented in Fig. 6.

Quadratic distance. The quadratic distance takes into account the color similarity between the histogram bins by using the symmetrical similarity matrix $A$. The matrix 


\begin{tabular}{|c|c|c|c|c|c|c|c|c|}
\hline$a_{11}$ & $a_{12}$ & $a_{13}$ & $a_{14}$ & $a_{15}$ & ....... & $\mathrm{a}_{\ln 2} 2$ & $\mathbf{a}_{\mathrm{nn}-\mathrm{I}}$ & $\mathrm{a}_{\ln }$ \\
\hline$a_{21}$ & $\mathrm{a}_{22}$ & $a_{23}$ & $a_{24}$ & $a_{25}$ & $\ldots \ldots$. & $a_{2(n-2)}$ & $\mathrm{a}_{2(\mathrm{n}-1)}$ & $a_{2 n}$ \\
\hline$a_{i 1}$ & $a_{i 2}$ & $a_{13}$ & $a_{i 4}$ & $a_{i 5}$ & $\ldots \ldots$ & $\mathrm{a}_{(\mathbf{i} \mathrm{n}-2)}$ & $a_{(p-1)}$ & $\mathrm{a}_{\mathrm{in}}$ \\
\hline$a_{\mathrm{m} 1}$ & $\mathrm{a}_{\mathrm{m} 2}$ & $a_{m 3}$ & $a_{\mathrm{m} 4}$ & $\mathrm{a}_{\mathrm{m5}}$ & $\ldots \ldots$ & $\mathrm{a}_{\mathrm{m}(\mathrm{m}(\mathrm{n} 2)}$ & $a_{\mathrm{m}(\mathrm{n}(\mathrm{n}-1)}$ & $\mathrm{a}_{\mathrm{mn}}$ \\
\hline & & & & & \multicolumn{4}{|c|}{$\begin{array}{c}C_{l_{j}} \text { is the } j^{\text {th }} \text { element of the centroid } \mathrm{l} \text {, } \\
\qquad c_{\mathrm{lij}}=\sum_{\mathrm{i}=1, \mathrm{~m}} \mathrm{a}_{\mathrm{ij}} / \mathrm{m}\end{array}$} \\
\hline$c_{11}$ & $\mathrm{c}_{12}$ & $c_{13}$ & $\mathrm{c}_{14}$ & $c_{15}$ & $\ldots \ldots$ & $\mathrm{c}_{(\mathrm{ln}-2)}$ & $\mathrm{c}_{(\mathfrak{l n}-1)}$ & $\mathrm{c}_{\mathrm{ln}}$ \\
\hline
\end{tabular}

Fig. 6. Centroid vector.

weights are normalized to obtain $0 \leq a_{p q} \leq 1$. So, the matrix diagonal is equal to 1 since any color is identical with itself $\left(a_{p p}=1\right)$. A coefficients $\left(a_{p q}\right)$ close to 0 represents dissimilarity between $p$ and $q$ bins. For example, in QBIC [8], the quadratic distance between two color histograms is used with a similarity matrix $A$ whose elements are defined by: $a_{i j}=\left(1-d_{i j} / d_{\max }\right)$, with $d_{\max }=\max _{i j}\left(d_{i j}\right), d_{i j}$ being Euclidean distance between the colors $i$ and $j$ in any color space. The two distributions, $H$ and $I$, may also be normalized in order that $1 \geq h_{c p}, i_{c p} \geq 0$, and $\Sigma_{p} h_{c_{p}=} \Sigma_{p} i_{c_{p}}=1$;

$$
D_{L 2}=\sqrt{ }\left(\Sigma_{l=1, n}\left(h_{c l}-i_{c l}\right)^{2}\right) \text {. }
$$

The quadratic distance $D_{Q}(H, I)=\sqrt{ }\left((H-I)\right.$.A. $\left.(H-I)^{T}\right)$ with $A$ the similarity matrix $(n \times n), A=\left[a_{p q}\right], a_{p q}$ weight of the similarity between the $p$ and $q$ bins. This distance makes it possible to obtain satisfactory results since it appreciates color similarity correctly. However, its major drawback is that it is time-consuming compared to the other distances. Euclidean distance results from the quadratic distance where $A$ matrix is the identity matrix (no correlation between the histogram bins).

For the texture, we extend the Euclidean distance to Fourier coefficients. Thus, the matching distance between the Fourier descriptors of texture $t^{\prime}$ of an image image ${ }^{\prime}$ and the Fourier descriptors of the texture $t$ of an image "image," is triggered by computing the distance between $t$ and $t^{\prime}$, namely:

$$
d\left(t, t^{\prime}\right)=\sqrt{ }\left(\Sigma_{n=1, N}\left(\left|T_{n}^{\prime}-K \cdot\right| T_{n} \mid\right)^{2}\right), N=10,
$$

for $t$ and $\mathrm{t}^{\prime}$ textures, we have a positive constant $K$, and for any $n \neq 0,\left|\mathrm{~T}_{n}^{\prime}\right|=K *\left|T_{n}\right|$, where

$$
Z_{n}=\sqrt{ }\left(\left|X_{n}\right|^{2}+\left|Y_{n}\right|^{2}\right)=\sqrt{ }\left(a_{n}{ }^{2}+b_{n}{ }^{2}+c_{n}{ }^{2}+d_{n}{ }^{2}\right) .
$$

That is to say, the textures are identical except for one geometric transformation. The translation, scale, and rotation have no effect on the module of Fourier coefficients. $K=1 / N *\left(\Sigma_{n=1, N}\left(\left|T^{\prime} n\right| /\left|T_{n}\right|\right)\right)$ is an estimation of $K$ which minimizes the error on the first $N$ (e.g., 11) coefficients of Fourier.

Entropy. The entropy is a statistical measure of the degree of cluster dispersions. In information theory, the entropy $\mathrm{S}=-\Sigma_{i=1, L}$ pi ln pi. pi = probability of the appearance of the feature of the centroid $i . \Sigma_{i} p i=1$. It is used by the algorithm in order insure that centroids are far from each other. The implementation of the formula presented, above is very time consuming. So, we approximate it, in the algorithm, by a simple procedure. This procedure considers that the entropy is proportional to the distribution homogenous of the features in the clusters. So, when we obtain clusters for which the cardinalities are not far from each other, then we consider that the entropy is high. However, when we obtain clusters, for which the cardinalities are so different, then we consider that the entropy is weak, and then the clustering result is not good. In this case, certain clusters contain a high number of features, and others contain a low number of features. For example, a cluster contains 2,000 features, and another one contains five features. So, we can say that the entropy is not good. In each iteration of the algorithm, the cluster that contains the highest number of features is splitting, and the cluster that contains the lowest number of features is deleted.

Distortion. The distortion is a statistical measure and a cost function that is used, by the algorithm, to estimate the degree of clustering around the centroids. An objective of the algorithm is to minimize this cost function. Distortion

$$
D=1 / T\left(\Sigma_{t=1, L} \Sigma_{k=1, \operatorname{cardinality}(Y t)} \operatorname{distance}\left(c_{t}, y_{k}\right)\right), y_{k} \in Y_{t} .
$$

$\mathrm{L}=$ number of clusters. $\mathrm{T}$ is the number of features of image databases. $c_{t}$ is the centroid of the cluster $Y_{t}$. In Fig. 7, Distortion(Cluster 3$) \approx$ Distortion(Cluster 4$)$ means that images of Cluster 3 and Cluster 4 are clustered with a similar degree around their gravity centers.

$$
\text { Distortion(cluster } 1)<\text { Distortion (Cluster } 2)
$$

means that the clustering degree of images in Cluster 1 is better than the clustering degree of images in Cluster2. Entropy

$$
(\{\text { Cluster } 1, \text { Cluster } 2\})>\operatorname{Entropy}(\{\text { Cluster } 3, \text { Cluster } 4\})
$$

means that the distributions of images around the gravity centers of Cluster 1 and Cluster 2 are more homogeneous than the distributions of images around the gravity centers of Cluster 3 and Cluster4. We note that Cluster3 contains five features and Cluster4 19 features.

Euclidean distance. The Euclidean distance $d$ between two vectors $v$ and $u$ is equal to $\sqrt{ }\left(\Sigma_{l=1, n}\left(v_{c l}-u_{c l}\right)^{2}\right)$. It is one of the most popular distances used in the clustering techniques.

\subsection{Algorithm}

Five steps distinguish the algorithm:

1. For both color and texture features, $L$ features are selected randomly from the learning set $L_{S}$. The learning set $L_{S}$ constitutes the set of features of all images of repositories. These $L$ features, selected 


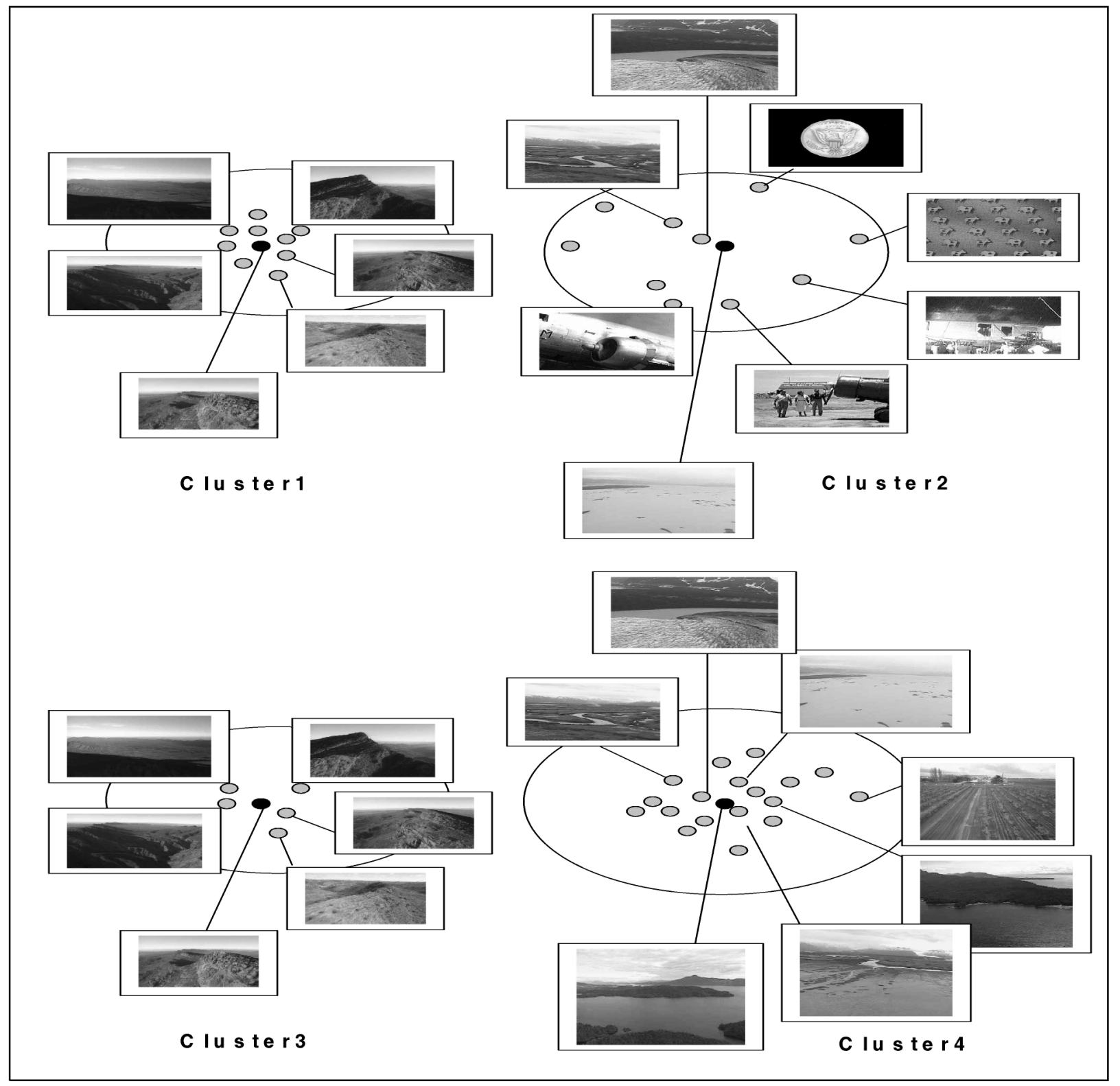

Fig. 7. Distortion and entropy computing.

randomly, constitute the initial visual dictionary $Y_{0}$. We consider that length $\left(Y_{0}\right)=L$ and length $\left(L_{S}\right)=T$. In our experiment, $L=252$ and $T=27,331$. So, length $\left(Y_{0}\right)=256$ and length $\left(L_{S}\right)=27,331$. It means that the learning set $L_{S}$ contains 27,331 colors and 27,331 textures, and the visual dictionary contains 256 distinct colors and 256 distinct textures. More generally, we consider $L \ll T$.

If the feature $=$ color, then the learning set $=$ $\left\{H_{1}, H_{2}, H_{3}, \ldots H_{T}\right\}$, a set of $T$ histograms. If the feature =texture, then the learning set $=\left\{S 1, S 2, \ldots, S_{T}\right\}$, a set of $T$ sequences of Fourier coefficients

$$
S i=((a i, b i),(c i, d i)) .
$$

2. At this step, we are in the first iteration $s$. To limit the number of iteration, $s$ is less or equal than $L / 2$, $(s \leq \delta=L / 2)$. So, the algorithm stops at $\delta=L / 2$. We suppose that at the iteration $s=(\delta=L / 2)$ the entropy is maximal.

Initialization: $D_{0}=\operatorname{Distortion}\left(Y_{0}\right) ; s=0 ;$ Custer $_{0}=$ $\left\{\right.$ Cluster $\left._{0, k} ; k=1, \ldots, L\right\}$

While $(\mathrm{s} \leq \delta)$ \{

3. $\quad /^{*}$ The features of the learning set $L_{S}$ are grouped around the visual dictionary features. The gravity centers are computed, and replaced by the most similar features of the suitable clusters. This operation also characterizes $k$-medoid algorithms [15]. The replacement of the gravity centers by the most similar image features will avoid the empty clusters.* /

Do \{ 
- $\quad s^{\prime}=0 ; s^{\prime}$ indicates the second iteration inside the first one.

- $\quad$ Based on the visual dictionary $Y_{s^{\prime}}=\left\{Y_{s^{\prime}, k} k=\right.$ $1, \ldots, L\}$ and the learning set $L_{S}$; we create, at the iteration $\mathrm{s}^{\prime}$, L clusters

$$
\left\{\text { Cluster }_{s^{\prime}, k} ; k=1, \ldots, L\right\},
$$

in which $\forall x \in L_{S}, \exists k$ unique, $k \in[1-L]$ and $x \in$ Cluster $_{s^{\prime}, k}$, and distance $\left(x, y_{k}\right)$ is minimal. So, if $x_{t} \in$ Cluster $_{s^{\prime}, k}$, then

$$
\operatorname{distance}\left(x_{t}, y_{k}\right) \leq \operatorname{distance}\left(x_{t}, y_{j}\right) \forall j \neq k \text {. }
$$

If the feature = texture, then distance $=$ Euclidean distance, else (feature $=$ color $)$, then distance $=$ quadratic distance.

Creation of the visual dictionary $Y_{s^{\prime}+1}$ and deletion of the visual dictionary $Y_{s^{\prime}}$.

$$
\begin{aligned}
Y_{s^{\prime}+1}= & \left\{\text { centroid } \text { Cluster }_{s^{\prime}, k}\right) k=1, \ldots, L ; \\
& \text { centroid }\left(\text { Cluster }_{s^{\prime}, k}\right)
\end{aligned}
$$

corresponds to the gravity center of the

$$
\text { Cluster } \left._{s^{\prime}, k} \cdot\right\} \text { Until }\left(D_{s^{\prime}-1}-D_{s^{\prime}} / D_{s}^{\prime}<\epsilon\right.
$$

$\rho^{*}$ The algorithm is reiterated in the new visual dictionary in order to obtain a new partition. The algorithm converges to a stable state by developing at each iteration the distortion criteria. Each iteration of the algorithm should reduce the distortion. So, $D_{s-1} \geq D_{s}$. The algorithm is stopped when the stability of distortion is achieved. The choice of the initial visual dictionary will influence the clusters obtained. The creation of the initial visual dictionary will be certainly performed by using the splitting technique [10]. */

$$
\}
$$

4. $\quad \quad^{*}$ To increase the entropy of the visual dictionary features, the highest apparition probability cluster is split into two subclusters, and the same clustering approach is applied to the two subclusters. * /

Splitting of a centroid of the visual dictionary $Y_{s-1}$ that supports the highest apparition probability $p_{i} . p_{i}$ corresponds to Cluster Cli $_{i}$ that has the maximum number of features. The centroid corresponds to the gravity center of the Cluster $_{s, i} \cdot\left(Y_{s-1, i^{\prime}}, Y_{s-1, i^{\prime \prime}}\right)=\operatorname{splitting}\left(Y_{s-1, i}\right)$.

- Deleting the centroid of the visual dictionary $Y_{s-1}$ that supports the lowest apparition probability $p_{j}$. $p_{j}$ corresponds to the Cluster ${ }_{s, j}$ that has the minimum number of features. The centroid, in question here, of the visual dictionary corresponds to the gravity center of the Cluster $_{s, j}$.

- Each splitting is followed by a deletion, so the cardinal of the visual dictionary remains constant (equal to $L$ ). In other words, the splitting step decomposes features $Y_{k}$ of the visual dictionary into two different features $Y_{k-\epsilon}$ and $Y_{k+\epsilon}$, where $\epsilon$ is a random vector of weak energy, and its distortion depends on the distortion of the split vector. The splitting step reduces not only the dependency between the initial visual dictionary and the final visual dictionary, but also increases the entropy of the visual dictionary. It means that, the visual features are grouped homogeneously around the features of the visual dictionary.

5. The algorithm is then applied to the new visual dictionary in order to optimize the produced features. So, the step 3 is applied to $Y_{s-1, i}$, and $Y_{s-1, i^{\prime \prime}}$, by considering the learning $=Y_{s-1, i}$, and then, the step 3 is applied on the whole visual dictionary that includes $Y_{s-1, i}$, and $Y_{s-1, i^{\prime}}$.

\}

The experimental results have shown that the distortion values decrease quickly as the splitting number rises. After a quick initial decrease, the distortion values decrease very slowly. Conversely, the entropy increases quickly as the number of splitting rises, and then, it increases very slowly.

\section{AsSOCIATION EXTRACTION}

Based on the visual dictionary, the approach discovers associations. Associations are knowledge shared by images of the same repositories (Birds, Animals, Aerospace, Cliffs, etc.).

The extraction of associations is elaborated in two steps: symbolic clustering and association discovering.

\subsection{Symbolic Clustering}

In the first step, the description of the content of images is transformed into a symbolic form defined in the visual dictionary. The most similar features defined in the visual dictionary replace the features of the image. Each feature of the visual dictionary is associated to a symbolic representation in order to manipulate it easily. In fact, in our experimentation, a vector of 256 bins implements a color feature. The texture feature is implemented by a fourdimension vector of 42 elements. So, manipulating a symbol is more convenient than such vectors and, significantly, simplifies the extraction process. For example, in the figure presented below (Fig. 8), the image is composed of region1 and region 2. Region1 is characterized by light red color and bird texture, and region 2 by watercolor and water texture.

A simple string does not describe light red color, but it is described by a color histogram. Even if the colors in regions of different images of the same repository, as presented in the following figure, are similar (i.e., light red), the histograms (numerical representation of color) associated with them are not generally identical; they may differ by only a few pixels.

That is why the visual dictionary has been created. It clusters similar colors and textures together in a symbolic form (Fig. 9). So, the histograms of colors and the series of Fourier coefficients of the texture associated to region 1 and region 2 are replaced by the most similar color and texture of 


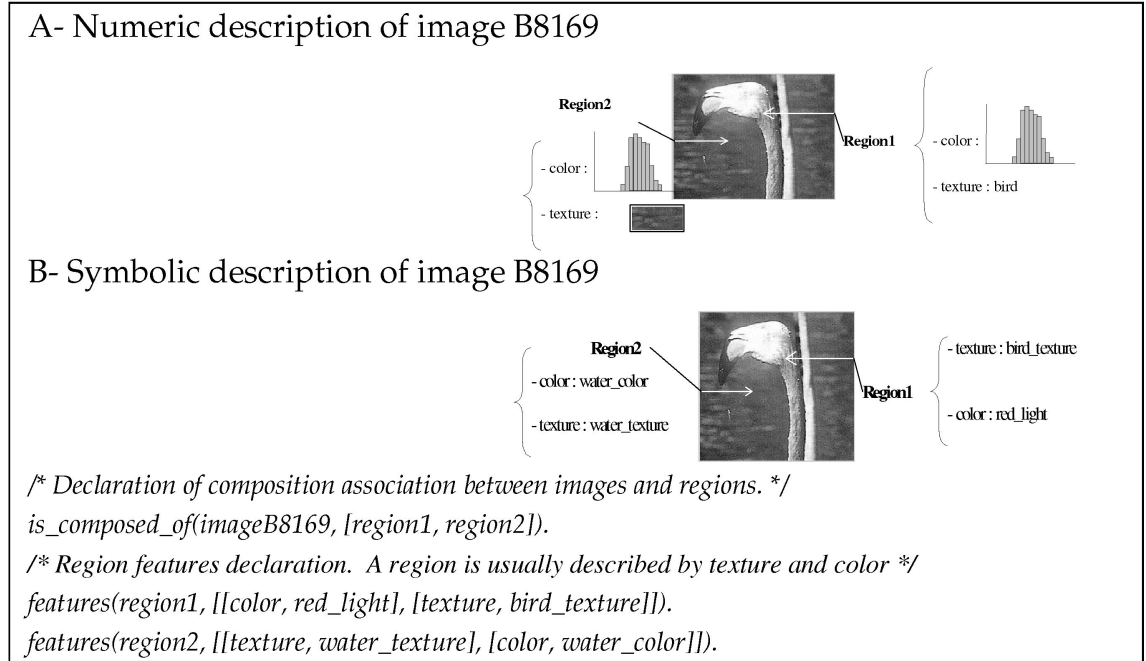

Fig. 8. Image symbolic representation.

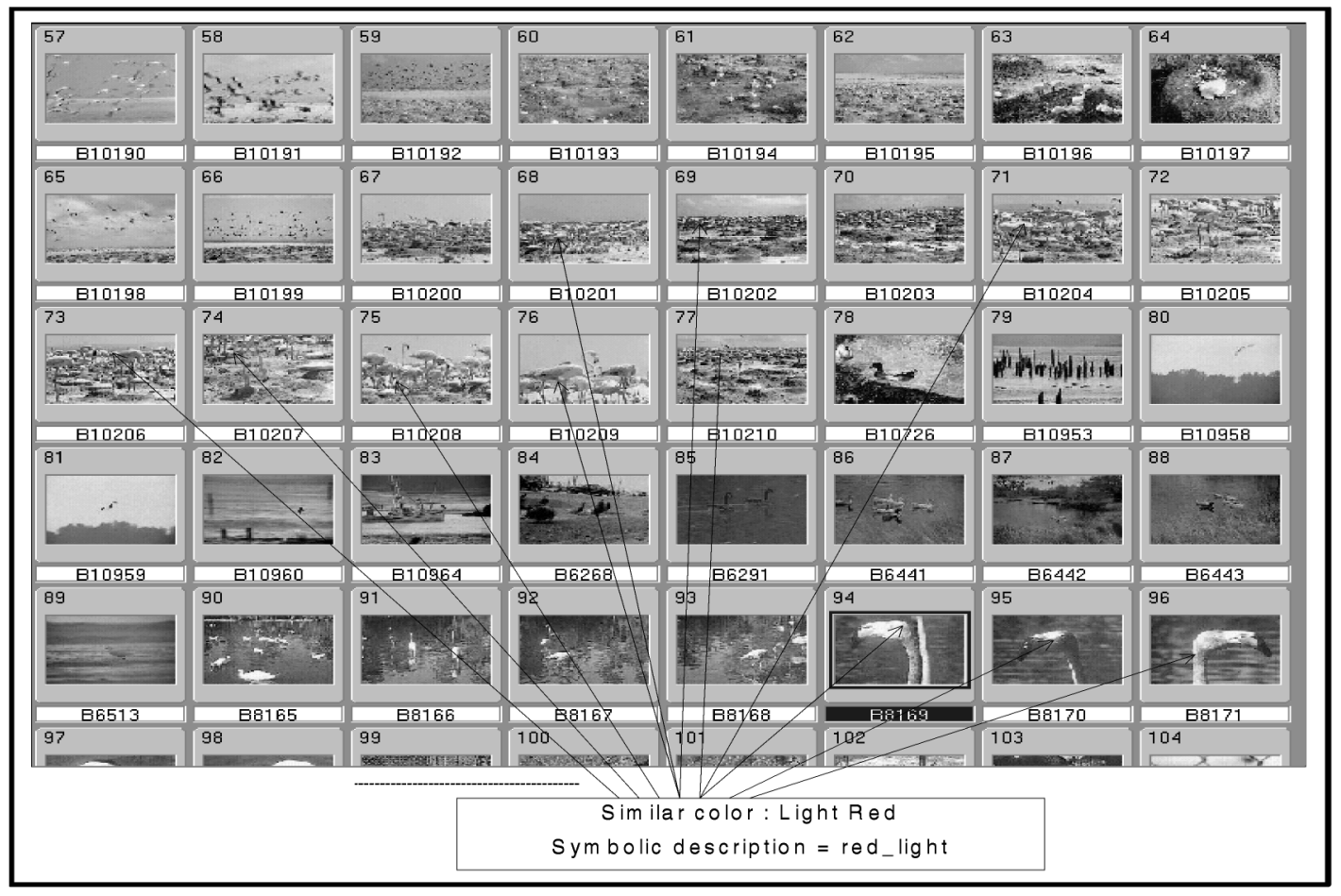

Fig. 9. These images belong to the repository: Bird. In this example, the light red color zones in the different images are grouped together in the symbolic form red_light because they are similar. Watercolor is not clustered in red_light because the distance between them is not the shortest one. However, it is clustered in the symbolic form water_color shared with other images. In the same way and based on appropriate distances, the system clusters, respectively, similar textures together in a symbolic form.

the visual dictionary. To simplify the manipulation of the visual dictionary features, the system uses symbolic representations: "red_light," "water_color," "bird_texture," "water_texture." Based on these descriptions, the discovery engine is triggered to discover the shared knowledge in the form of associations, and this constitutes the second step of the association extraction.

\subsection{Association Discovery}

In the second step, the knowledge discovery engine automatically determines common associations among the image features. These associations are in the form of
Premise $=>$ Conclusion with a confidence (Fig. 10). These associations are called statistical as they accept counterexamples.

Here is the general principle of the association discovery engine:

\#define ConditionalProbability = Real [0-1];

\#define ImplicationIntensity = Real [0-1];

\#define Confidence $=($ ConditionalProbability,

ImplicationIntensity);

\#define Association $=(\{$ Color, Texture $\} \times\{$ Color, Texture $\} \times$ Confidence) 


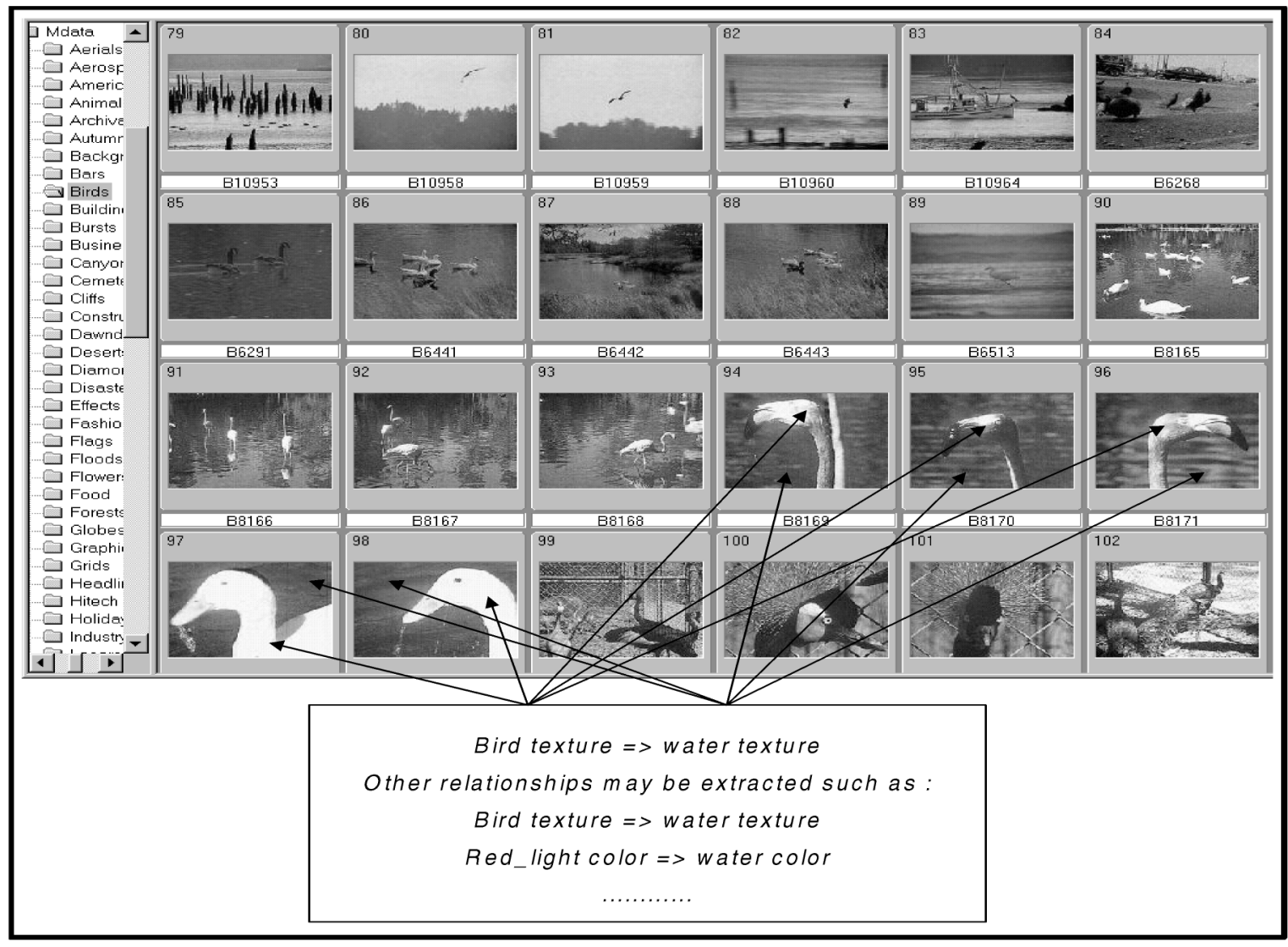

Fig. 10. Samples of extracted rules by the knowledge discovery engine.

Color $=$ histogram [256];

Texture = fourier [10] [10];

association_local set-of Association $=\varnothing ;$

association_global set-of Association $=\varnothing$;

Principle of the association discovery

engine (image_repository $y_{k}$ image_collection)

\{

${ }^{*} T$ is the length of image repository */

For $(i=0, i<T, i++)$

\{

* extraction of associations between features of

the whole image + extraction of all associations

between features of the regions of the images, if

the regions have been identified */

association_local $=$

image $i$.extract_all_binary_associations(image_repository ${ }_{k}$

image_collection);

association_local $=($ association_local -(

association_global $\cap$ association_local ) );

For $(j=0, j<$ length(association_local),$j++)$

\{association_local(j).ConfidenceMeasure ();

* Confidence measure $=$ conditional probability measure and implication intensity measure. */

\}

association_global $=$ association_global $\cup$

(association_local - (association_global $\cap$ association_local ))
\} association_global = association_global $-\{a \in$ Association, ConditionalProbabilityConfidence $(a)<95 \%$ and ImplicationIntensityConfidence $(a)<90 \%\}$ \}

Confidence is very important in order to estimate the quality of the associations induced. In order to estimate the confidence of associations, we implement two confidence measures: the conditional probability and the implication intensity. The user should indicate the threshold above which associations discovered would be kept (relevant associations). In fact, the weak associations are associations that are not representative of the shared knowledge.

The conditional probability of the association $a=>b$ answers the following question: "what are the chances of proposition $b$ being true when proposition $a$ is true? The definition of this measure is given in the following figure (Fig. 11).

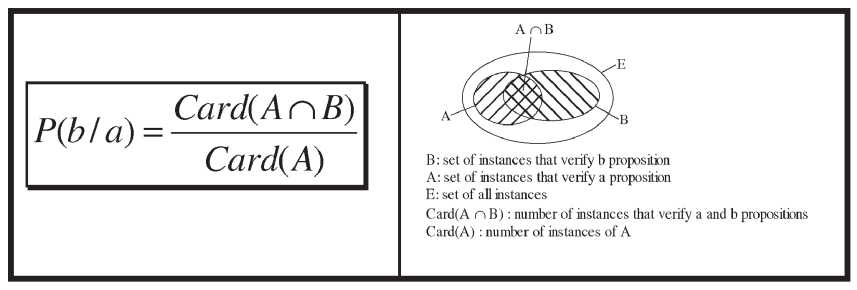

Fig. 11. Conditional probability formula. 


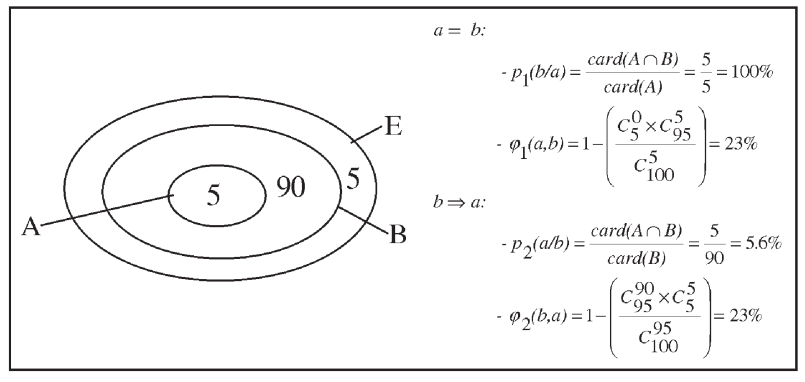

Fig. 12. Association accuracy example.

More intuitively, conditional probability allows us to estimate the confidence of associations, considering the number of counterexamples. If associations have the conditional probability equal to 5.6 percent, then the associations have lots of counter examples. In $E$ (universe set), there are many objects that belong to $B$, but not to $A$. If the associations have the conditional probability equal to 100 percent, then they have no counterexample. So, objects that respect proposition $a$, also respect proposition $b$.

Conditional probability allows the system to determine the discriminating characteristics of considered images. However, this measure presents several drawbacks. That is why we also implemented the intensity of implication [9]. For example, implication intensity requires a certain number of examples or counterexamples. When the doubt area is reached, the intensity value increases or decreases rapidly contrary to the conditional probability that is linear. In fact, implication intensity simulates human behavior better than other confidence measures and particularly conditional probability.

Moreover, implication intensity increases with the considered population sample covering. The considered sample must be large enough in order to draw relevant conclusions. Finally, implication intensity takes into consideration the sizes of sets and, consequently, their influence. The figure presented bellow illustrates the latter point. $P_{1}$ (100 percent)

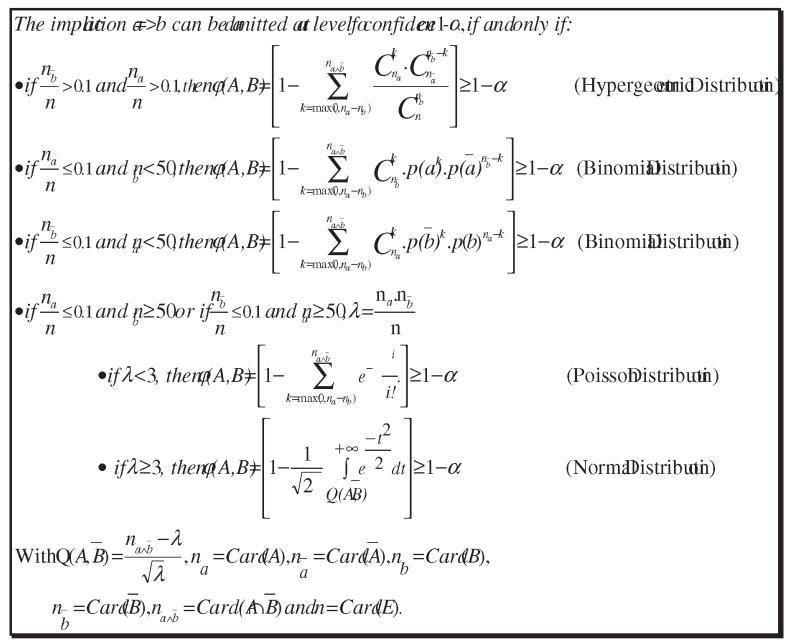

Fig. 13. Implication intensity formulas.
TABLE 1

Extracted Associations

\begin{tabular}{|c|c|c|c|}
\hline \multicolumn{4}{|c|}{$\begin{array}{l}\text { KNOWLEDGE DISCOVERY RESULTS } \\
\text { WITH THE CONDITIONAL PROBABILITY (CP) } \\
\text { AND IMPLICATION INTENSITY (II) FORMULAS }\end{array}$} \\
\hline \multicolumn{4}{|c|}{$\begin{array}{l}\text { The threshold is } 62 \% \text {. } \\
\text { Extracted associations are by decreased order of relevance: }\end{array}$} \\
\hline $\begin{array}{c}\text { Association } \\
\text { Number }\end{array}$ & Extracted Associations & \begin{tabular}{|c|}
$\mathrm{CP}$ \\
Value \\
in $\%$
\end{tabular} & $\begin{array}{l}\text { II Value } \\
\text { in } \%\end{array}$ \\
\hline 1 & (texture, texture 2$) \Rightarrow$ (color, color 3$)$ & 100 & 96.08 \\
\hline 2 & (color, color 3$) \Rightarrow($ texture, texture 2$)$ & 66.66 & 91.62 \\
\hline 3 & (texture, texture 1$) \Rightarrow($ color, color 1$)$ & 100 & 87.4327 \\
\hline 4 & (texture, texture6) $\Rightarrow$ (color, color 3$)$ & 100 & 78.8928 \\
\hline 5 & (texture, texture 3$) \Rightarrow($ color, color 1$)$ & 100 & 64.5496 \\
\hline 6 & (texture, texture 4$) \Rightarrow($ color, color 1$)$ & 100 & 64.5496 \\
\hline 7 & (texture, texture5) $\Rightarrow$ (color, color 1$)$ & 100 & 64.5496 \\
\hline 8 & (texture, texture 6$) \Rightarrow$ (color, color 1$)$ & 100 & 40.4598 \\
\hline
\end{tabular}

and $\varphi_{1}$ (23 percent) values are very different because conditional probability does not take into consideration the fact that proposition $b$ is verified by many objects. On the contrary, implication intensity considers that it is not surprising that an object of $A$ verifies proposition $b$ because many objects of the considered sample verify proposition $b$.

The figures (Fig. 12 and Fig. 13) explain the implication intensity. Let $A, B$, and $E$ be, respectively, the sets of instances that verify proposition $a$, the set of instances that verify proposition $b$, and the set of all instances or the universe. From a theoretical point of view, implication intensity measures the degree of statistical unexpectedness of size $A \cap \bar{B}$ (this set contains objects that verify proposition $a$ and that do not verify proposition $b$ considering the sizes of

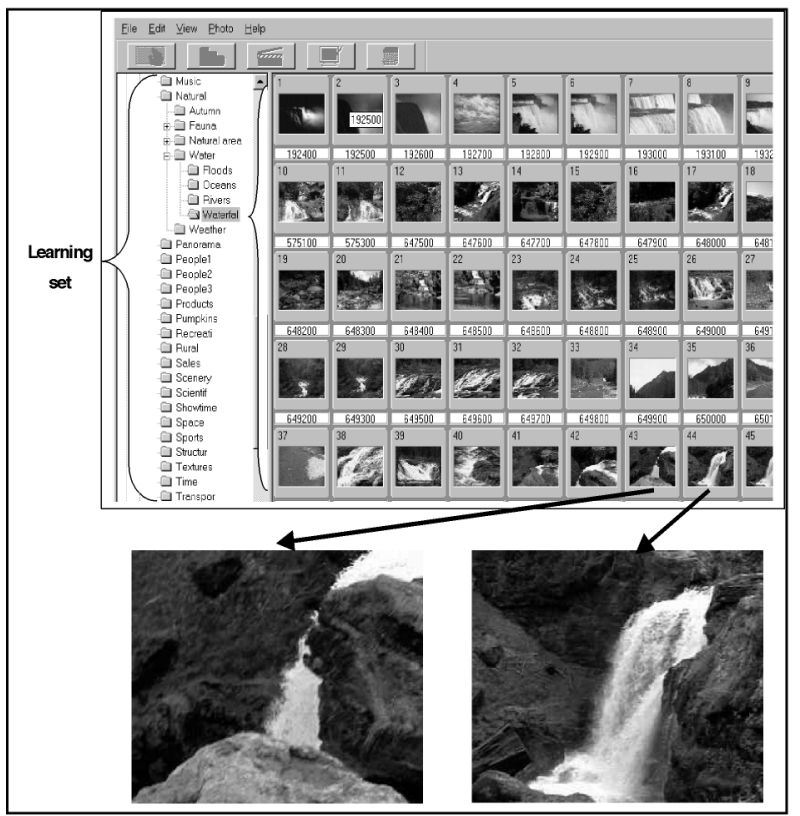

Fig. 14. A sample of the learning set. It concerns waterfalls. 
TABLE 2

Summary of the Experimentation Repositories

\begin{tabular}{|c|c|c|}
\hline Repositories & $\begin{array}{l}\text { Numbe } \\
\text { rof } \\
\text { images }\end{array}$ & Sub-repositories \\
\hline Animals & 1008 & $\begin{array}{l}\text { Various birds, crustaceans, such as } \\
\text { crabs, lobsters, shrimp, shellfish, } \\
\text { various fish, various insects, various } \\
\text { mammals, micro organisms and } \\
\text { small invertebrates }\end{array}$ \\
\hline Food & 135 & $\begin{array}{l}\text { Prepared food, raw fruits and } \\
\text { vegetables }\end{array}$ \\
\hline $\begin{array}{l}\text { Holidays and } \\
\text { special } \\
\text { occasions } \\
\end{array}$ & 693 & $\begin{array}{l}\text { Holidays other than Christmas, } \\
\text { Christmas }\end{array}$ \\
\hline People & 9031 & $\begin{array}{l}\text { People participating in sports, } \\
\text { business and occupations, families } \\
\text { and children, leisure activities, } \\
\text { political figures and events, public } \\
\text { servants, fashion portraits and close- } \\
\text { ups }\end{array}$ \\
\hline Plants & 527 & $\begin{array}{l}\text { Flowers, bouquets and floral } \\
\text { arrangements, gardens, trees and } \\
\text { leaves }\end{array}$ \\
\hline Religion & 55 & $\begin{array}{l}\text { Churches, religious ceremonies, } \\
\text { religious monuments, religious } \\
\text { objects }\end{array}$ \\
\hline Scenic & 3629 & $\begin{array}{l}\text { Deserts and canyons, mountains and } \\
\text { fields, ocean scenes, skies and clouds, } \\
\text { water scenes, such as waterfalls } \\
\text { (Figure 14), lakes, mountains, sunsets }\end{array}$ \\
\hline Structures & 2238 & $\begin{array}{l}\text { Miscellaneous architectural objects, } \\
\text { commercial buildings, residential } \\
\text { buildings, bridges, city scopes, } \\
\text { architectural monuments }\end{array}$ \\
\hline $\begin{array}{l}\text { Technical and } \\
\text { educational }\end{array}$ & 1524 & Largely science and space \\
\hline $\begin{array}{l}\text { Transportatio } \\
\mathrm{n}\end{array}$ & 2800 & $\begin{array}{l}\text { Cars, watercraft, motorcycles, hot air } \\
\text { balloons, three-wheelers, ..., aircraft, } \\
\text { seagoing vessels, trains }\end{array}$ \\
\hline Others & 222 & \\
\hline $\begin{array}{l}\text { Background } \\
\text { objects }\end{array}$ & 4189 & $\begin{array}{l}\text { Art objects, background fireworks, } \\
\text { colorful background patterns, } \\
\text { antiques, road-signs, comic signs, ..., } \\
\text { close-ups of object textures, close-ups } \\
\text { of clustered objects }\end{array}$ \\
\hline
\end{tabular}

$A, B$, and $E$ sets, and assuming there is no a priori link between $A$ and $B$. The objects of the database belonging to $A$ and $B$ determine the cardinals or the sizes of $A$ and $B$ subsets of $E$.

The knowledge discovery engine returns the associations in the form of Premise $=>$ Conclusion. The associations, whose intensity and conditional probability are greater than or equal to a certain threshold, are saved. This threshold is defined manually and depends of the application repositories.
TABLE 3

Experimental Results

\begin{tabular}{|c|c|c|c|c|}
\hline \multirow[t]{2}{*}{ Queries } & \multicolumn{2}{|c|}{$\begin{array}{l}\text { Knowledge-content-based } \\
\text { queries }\end{array}$} & \multicolumn{2}{|c|}{$\begin{array}{l}\text { Content-based- } \\
\text { queries }\end{array}$} \\
\hline & Precision & Recall & Precision & Recall \\
\hline Query 1 & $78 \%$ & $57 \%$ & $27 \%$ & $67 \%$ \\
\hline Query 2 & $63 \%$ & $66 \%$ & $56 \%$ & $51 \%$ \\
\hline Query 3 & $52 \%$ & $68 \%$ & $38 \%$ & $63 \%$ \\
\hline Query 4 & $63 \%$ & $62 \%$ & $49 \%$ & $56 \%$ \\
\hline ........ & & & & \\
\hline Query 49 & $82 \%$ & $52 \%$ & $37 \%$ & $59 \%$ \\
\hline Query 82 & $85 \%$ & $48 \%$ & $43 \%$ & $54 \%$ \\
\hline Average & $75 \%$ & $82 \%$ & $52 \%$ & $65 \%$ \\
\hline
\end{tabular}

Let us consider an example (Table 1) that presents associations discovered and qualified from art object repository. This set of induced associations corresponds to knowledge shared by the image repository. This knowledge is helpful for driving the image search and classification. The visual dictionary has been created on the basis of the algorithm presented previously. The repository contains a large number of art object images. After translating features of these images into symbolic form, the system discovers associations in which a sample is presented in Table 1.

Extracted associations are validated when the conditional probability and the association intensity are greater than specified values. For example, (texture,texture6) $\Rightarrow$ (color, color 1$)$ has 100 percent conditional probability and 40.4598 percent implication intensity. Texture 6 corresponds to an art object texture, and color color 1 corresponds to a gold object. Since the association intensity is less than 90 percent, the system will not save it. We explain this weak value of association intensity by the fact that there are few examples that respect this association.

(texture, texture 2$) \Rightarrow($ color, color 3 ) (association 1$)$ is the best association because the conditional probability value is 100 percent and the implication intensity is 96.08 percent. Texture 2 clusters African drum textures, and color 3 corresponds to a partial region of yellow color. So, during image insertion or image retrieval in the repositories, the classification of an image $X$ in the art object repository should, on the basis of the confidence, respect this association. This association means that when we have a texture that describes an African drum then we would have color 3 in an image $X$ region with a confidence equal to 100 percent for conditional probability and 96.08 percent for implication intensity.

In the retrieval task, when the user specifies an image (called source image) as the base of the query, and asks "find images similar to the source image," the system will not match the source image with all images of the database. It will match the source image features with all the target images of the suited repositories. The suited repositories 
contain associations globally respected by the source image. For example, if we have a source image that contains a "head" texture, but it does not globally verify the associations associated to this repository, we can deduce the weakness association between the source image and the repository. The system matches the source image with repositories through their learned and stored associations.

\section{8 "Efficiency" Evaluation}

We have conducted extensive experiments of varied data sets to measure the efficiency of the knowledge-contentbased query. First, we present the metrics used to measure retrieval performance followed by a description of the data sets. Finally, we present the results along with our observations.

\subsection{Evaluation Method}

The retrieval system can be evaluated by considering its capacity to effectively retrieve information relevant to a user. It is called the retrieval efficiency. Retrieval efficiency is measured by recall and precision metrics [20], [21]. For a given query and a given number of images retrieved, recall gives the ratio between the number of relevant images retrieved and the total number of relevant images in the collection considered. Precision gives the ratio between the number of relevant images retrieved and the number of retrieved images.

$$
\begin{aligned}
& \text { Precision }=\mid \text { relevant } \cap \text { results }|/| \text { results } \mid \\
& \text { Recall }=\mid \text { relevant } \cap \text { results }|/| \text { relevant } \mid .
\end{aligned}
$$

Recall and precision values for a system can be represented in a recall and precision graph [19], where the precision of the system is plotted as a function of the recall. This representation enables, for example, measurement of the precision at different recall points.

\subsection{Data Sets}

We have conducted experiments on a data set which is a collection of images covering a wide range of repositories including animals, panorama, archive, flowers, scenery, people, nature (e.g., Fig. 14), etc. This collection contains around 27,331 images and requires one Go for data storage, $110 \mathrm{MB}$ for metadata (index), and $36 \mathrm{MB}$ for index after compression.

The collection was handpicked to provide an assortment of breathtaking, high-quality photographs. Included are:

- 3,500 high-resolution photos, professional quality JPG photos at 240 DPI and 16 million of colors,

- 15,000 medium resolution 8 bit color photos (jpg), a stunning variety of photos and special effects,

- 7,500 black and white historical photo clips (jpg), amazing shots from memorable moments in history; it includes shots of people, nature, technology, sports, food, and more. Historical images portray movie stars, politicians, royalty, sports, heroes, memorable events, and everyday life.
- 1,331 for different resolutions and different semantics of images.

The above list (Table 2) describes the repositories and subrepositories of this collection.

All images are catalogued into broad repositories and each image carries an associated description. In this case, manually separating the collection into relevant and nonrelevant sets was unfeasible due to the size. It is not simple to obtain the exact number of positive results in the large image repositories. Instead, we estimate the cardinal of the result set of each query. The preclassification of images in semantic repositories such as panorama, flowers, etc. simplifies our estimation process. For certain images, we predetermine by hand and by exhaustive browsing a set of relevant image results. For other images, we start the search by issuing the query involving textual descriptions or visual features, to obtain the first set of image results. The set is refined and expanded by using query by example and exhaustive browsing.

\subsection{Results and Analysis}

The recall and precision values for our system are computed as follows: 82 reference ("query") images are selected from the test collection. The subset of images is selected per repository (waterfalls, fires, panorama, etc.). For an image reference, we associate a knowledge-content-based query and we associate a content-based query that doesn't use the knowledge associated to repositories. The threshold used to select multiple associations is set at 95 percent and 90 percent, respectively, for conditional probability and implication intensity confidences. The threshold $\epsilon$ used to determine the stability of the distortion mean is set at 0.05 for both local and global clustering. So, the local clustering algorithm is stopped when (distortion $D_{s-1}$ at iteration $s-1$ - distortion $D_{s}$ at iteration $s$ ) / distortion $D_{s}$ at iteration $s<\epsilon$. Note that in order to perform comprehensive examinations of the number of features in the visual dictionary, we purposely choose 256 color features and 256 texture features. So, the length of the visual dictionary $L$ is equal to 512 features. The $\delta$ offset used to determine the number of split is defaulted to $L / 2=256$ for texture and color.

Since it is not possible to retrieve all relevant images, our experiment evaluates only the first 50 ranked images. For experimental simplification, we don't use the similarity ranges. In the content-based queries, each query is decomposed into texture and color subqueries.

Judging from the table (Table 3 ) that summarizes results, it is obvious that the use of knowledge leads to improvements in both precision and recall over the majority of queries tested. The average improvements of knowledgecontent-based queries over content-based queries are 23 percent for precision and 17 percent for recall. Precision and recall are better for knowledge-content-based queries (queries that mix visual features and knowledge associated to repositories) than for queries that use only visual features (colors and textures). 

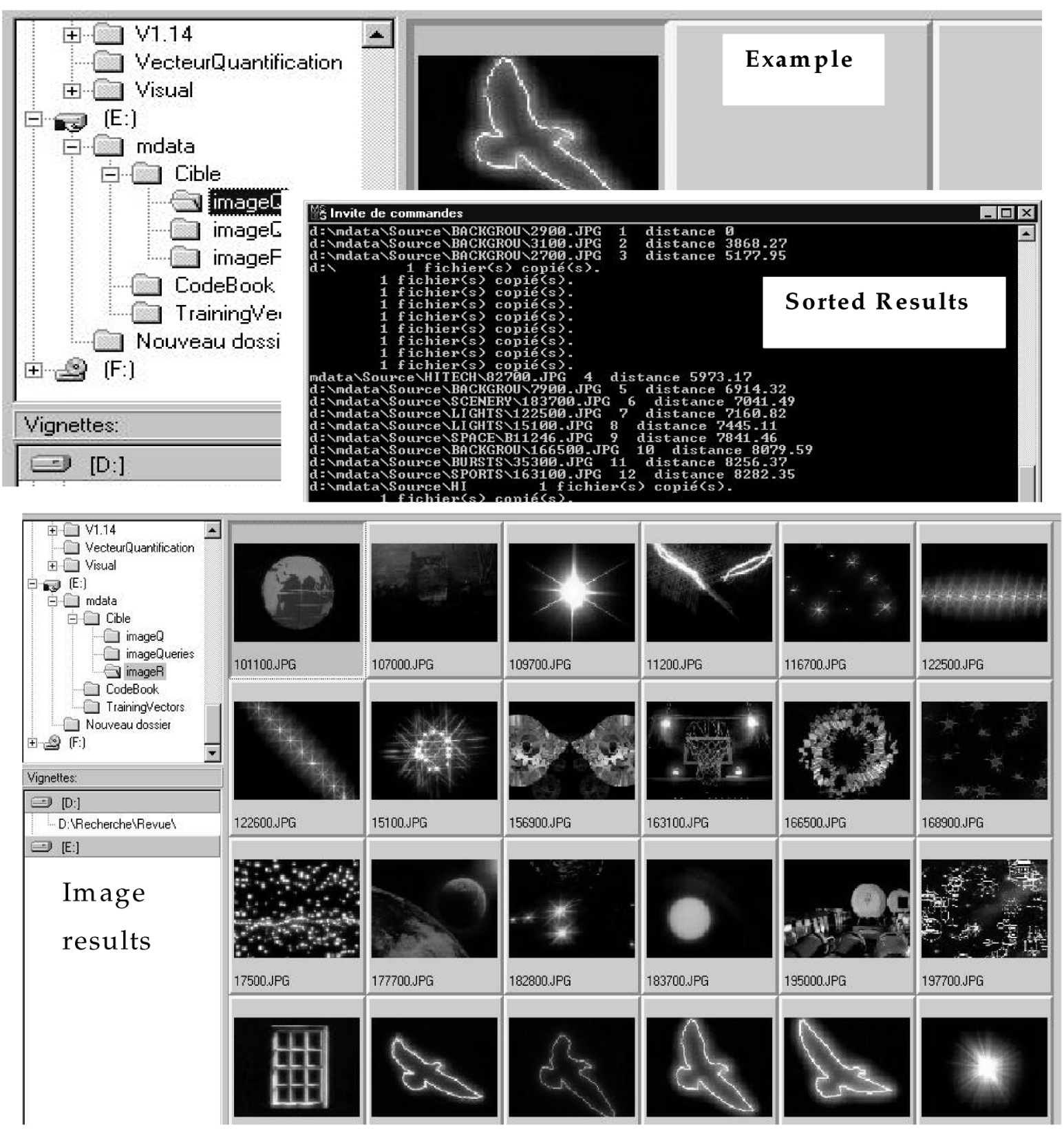

Fig. 15. "Find images that are similar to visual features specified by the user," the features are specified by the user through the image example.

We observe that, for a subset of images, the precision of content-based queries is better than the precision of knowledge-content-based queries. A possible explanation for this is that images of the same semantic repositories may have large variations with dissimilar visual descriptions (images of people, images of holidays, etc.), and images from different semantic repositories might share common visual features. So, knowledge-content-based retrieval that selects only one repository may forget images that are visually similar to the query examples, and belong, semantically, to several repositories.

We observed that the general principle of "the larger the retrieved set, the higher the recall, and the lower the precision" is observed.

\section{Conclusion}

The traditional content-based image indexing and retrieval architecture is composed of three important components: extraction, representation, and retrieval. Extraction and representation components constitute the heart of the architecture; together they constitute the indexing component. The extraction component extracts, automatically or semiautomatically, regions of images and computes features such as color and texture of the regions. The extracted features are represented as or transformed into suitable models and data structures, and then stored in a durable index. The retrieval component constitutes the eyes of the architecture. It searches images by selecting target images 


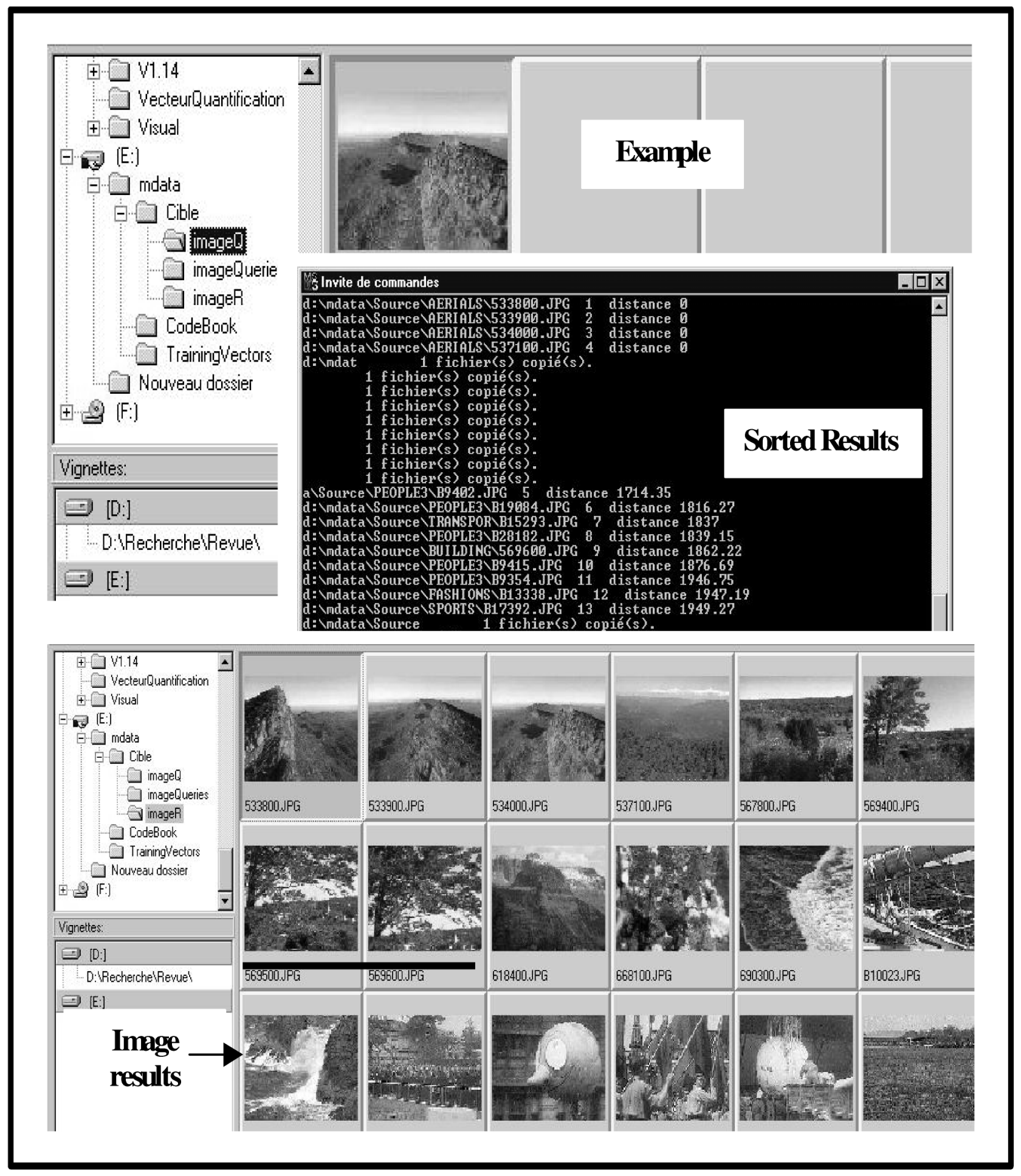

Fig. 16. "Find images that contain aerial panorama."

or content properties such as color, texture of image regions, or combinations of these. The retrieval process computes distances between source (example) and target features, and sorts the most similar images.

In this paper, we have presented the interest of knowledge discovery in the traditional architecture of the contentbased indexing and retrieval in large image repositories. The knowledge discovery engine extracts associations that characterize image repositories. From the features of images belonging to the same repository, the system finds the pattern of interest in the form of associations that are qualified on the basis of two confidence measures (conditional probability and implication intensity). These induced associations are very helpful for:

1. the comprehension of the considered repository,

2. the automatic classification of new images during their insertion in large repositories, 

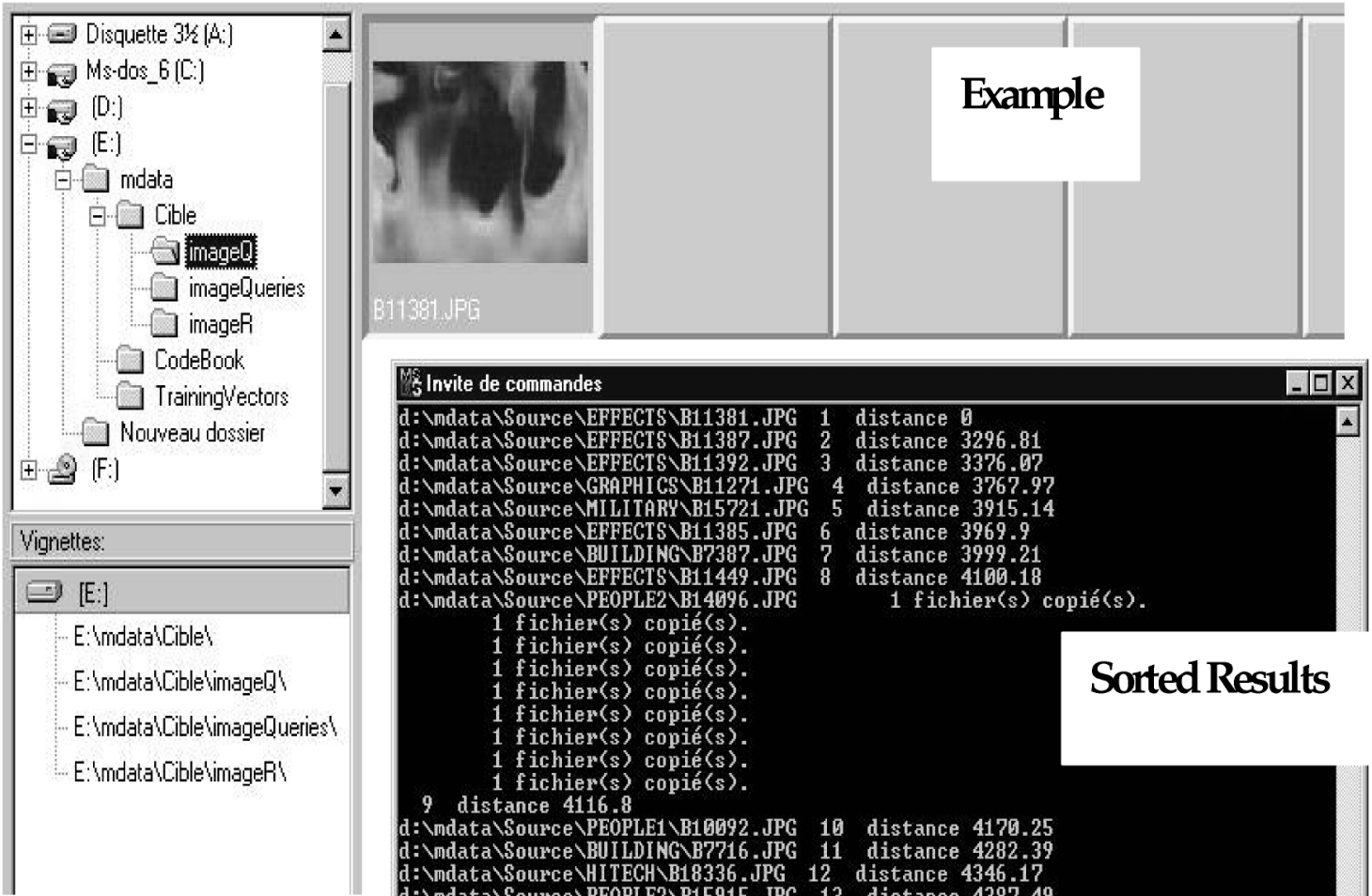

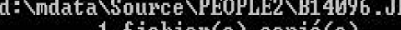

1 fichier $(s)$ copié(s).

1 fichier (s) copié(s).

1 fichier (s) copié(s).

1 fichier(s) copié(s).

1 fichier $(s)$ copié(s).

1 fichier (s) copié(s).

1 fichier $(s)$ copié(s).

distance 4116.8

d: \ndata $\backslash$ Source $\backslash$ PEOPLE1 \B10092.JPG 10 distance 4170.25

d: \mdata \ource $\backslash B U I L D I N G \backslash B 7716 . J P G ~ 11$ distance 4282.39

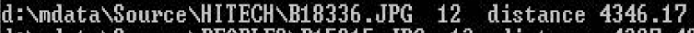

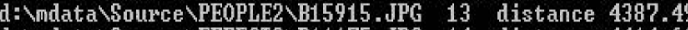

\section{Sorted Results}

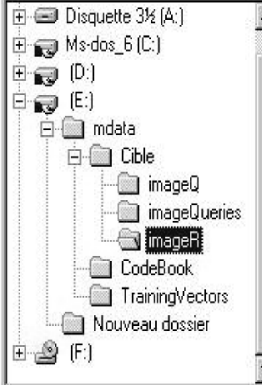

Wignettes:

E:'mdata'Cible'

E:'imdata'Cibletinaged?

E:'mdata'Cible'tinage[ueries'

E'Imdata'Cible'timageR'
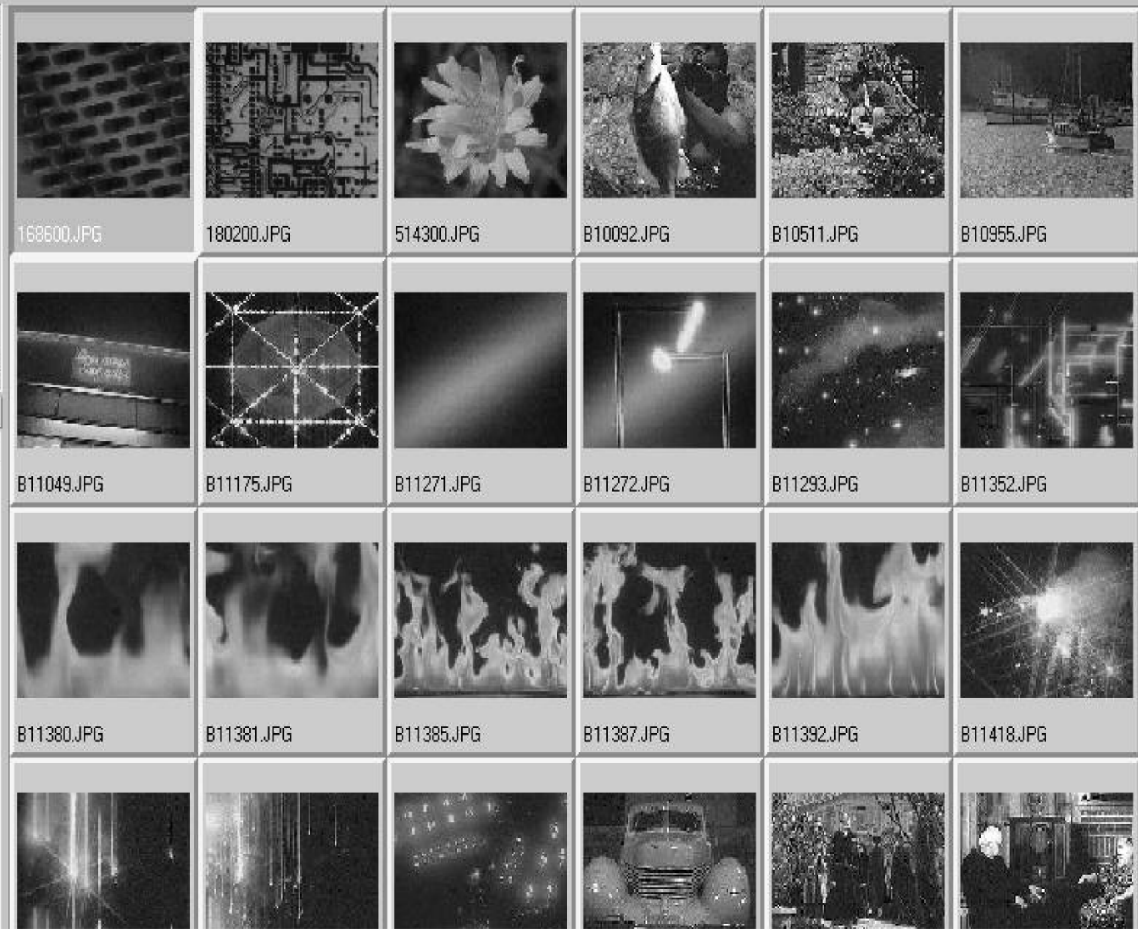

Fig. 17. "Find images that contain fire."

3. obtaining results with more semantics, and

4. improving the retrieval process.

More generally, we strongly believe that the discovery of hidden associations from multimedia will play in the future a revolutionary role in the content-based multimedia indexing and retrieval.

\section{APPENDIX}

\section{ANNEX: Query by EXAMPLES}

We present, here, a sample of "query by examples" returned by the knowledge-content-based retrieval system (Figs. 15, 16, 17). 


\section{ACKNOWLEDGMENTS}

The author would like to thank Frederic Andres for his valuable discussions.

\section{REFERENCES}

[1] S.F. Chang, J.R. Smith, M. Beigi, and A. Benitez, "Visual Information Retrieval from Large Distributed Online Repositories," Comm. ACM, vol. 40, no. 12, pp. 63-67, 1997.

[2] W. Chang, G. Sheikholeslami, J. Wang, and A. Zhang, "Data Resource Selection in Distributed Visual Information Systems," IEEE Trans. Knowledge and Data Eng., vol. 10, no. 6, pp. 926-946, Nov./Dec. 1998.

[3] T. Chua, K. Teo, B. Ooi, and K. Tan, "Using Domain Knowledge in Querying Image Databases," Multimedia Modeling, Towards the information Superhighway, 1996.

[4] P. Danzig, S. Li, and K. Obraczk, "Distributed Indexing for Autonomous Internet Services," technical report, Dept. of Computer Science, Univ. of South California, June 1992.

[5] S. Deerwester, S. Dumais, G. Furnas, T. Landauer, and R. Harshman, "Indexing by Latent Semantic Analysis," J. Am. Soc. Information Science, vol. 41, pp. 391-407, 1990.

[6] C. Djeraba and M. Bouet, "Digital Information Retrieval," Proc. ACM CIKM '97, Nov. 1997.

[7] C. Djeraba, M. Bouet, and H. Briand, "Concept-Based Query in Visual Information Systems," Proc. IEEE Advances in Digital Libraries, pp. 299-308, 1998.

[8] C. Faloutsos, M. Flickner, W. Niblack, D. Petrovic, W. Equitz, and R. Barber, "Efficient and Effective Query by Image Content," research report, IBM Alameda Research Center, 1993.

[9] R. Gras, "THE EISCAT CORRELATOR, EISCAT technical note," Kiiruna 1982, EISCAT Report 82/34, 1982.

[10] R.M. Gray, "Vector Quantization," IEEE ASSP Magazine, pp. 4-29, Apr. 1984.

[11] A. Gupta and R. Jain, "Visual Information Retrieval," Comm. ACM, vol. 40, no. 5, May 1997.

[12] J. Huang, R. Kumar, and R. Zabih, "An Automatic Hierarchical Image Classification Scheme," Proc. Sixth ACM Int'l Conf. Multimedia (ACM MM-98), 1998.

[13] R. Jain, "Content-Based Multimedia Information Management," Proc. Int'l Conf. Data Eng. (ICDE), pp. 252-253, 1998.

[14] B. Kahle and A. Medlar, "An Information System for Corporate Users: Wide Area Information Servers," ConneXions-The Interoperability Report, vol. 5, no. 11, Nov. 1991.

[15] L. Kaufman and P.J. Rousseeuw, Finding Groups in Data: An Introduction to Cluster Analysis. John Wiley \& Sons, 1990.

[16] M. Koster, "ALWEB: Archie-Like Indexing in the Web," Computer Networks and ISDN Systems, vol. 27, no. 2, pp. 175-182, 1994.

[17] Y. Linde, A. Buzo, and R.M. Gray, "An Algorithm for Vector Quantizer Design," IEEE Trans. Comm., vol. 28, no. 1, pp. 84-95, Jan. 1980.

[18] A. Pentland, R.W. Picard, and S. Sclaroff, "Photobook: Tools for Content-Based Manipulation of Image Databases," Proc. SPIE-94, pp. 34-47, 1994.

[19] V. Raghavan, G. Jung, and P. Bollman, "A Critical Investigation of Recall and Precision as Measures," ACM Trans. Information Systems, vol. 7, no. 3, pp. 205-229, July 1989.

[20] C.J. Keith van Rijsbergen, Information Retrieval, second ed. London: Butterworths, 1979.

[21] S. Gerard, Automatic Information Organization and Retrieval, chapter 4. McGraw Hill Book Co, New York, 1968.

[22] C.T. Zahn and R.Z. Roskies, "Fourier Descriptors for Plane Closed Curves," IEEE Trans. Computers, 1972.

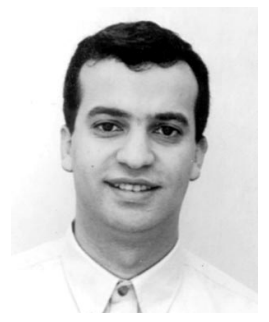

Chabane Djeraba received the BS degree from the National Institute of Computer Science (INI), Algiers, Algeria, in 1989, the MS degree in computer science from Pierre Mendes France University, Grenoble, France, in 1990, and the $\mathrm{PhD}$ degree in computer science from Claude Bernard University, Lyon, France, in 1993. In the spring of 1994, he joined the Polytechnic School at Nantes University, where he is now an associate professor. His research interests include multimedia mining, visual information systems, and multimedia synchronization. He has published several technical papers in these areas. He has served on the program committees of various conferences. Currently, he is on the editorial board of the Journal of Multimedia Tools and Applications, and member of the IEEE Computer Society and ACM.

$\triangleright$ For more information on this or any computing topic, please visit our Digital Library at http://computer.org/publications/dlib. 